\title{
Constraining quantum collapse inflationary models with current data: The semiclassical approach
}

\author{
María Pía Piccirilli垉, Gabriel León ${ }^{1}$, Susana J. Landau ${ }^{2}$, Micol Benetti ${ }^{345}$, and Daniel Sudarsky ${ }^{6}$ \\ ${ }^{1}$ Grupo de Astrofísica, Relatividad y Cosmología, \\ Facultad de Ciencias Astronómicas y Geofísicas, Universidad Nacional de La Plata, \\ Paseo del Bosque S/N 1900 La Plata, Pcia de Buenos Aires, Argentina. \\ ${ }^{2}$ Departamento de Física, Facultad de Ciencias Exactas y Naturales, \\ Universidad de Buenos Aires and IFIBA, CONICET, \\ Ciudad Universitaria - PabI, Buenos Aires 1428, Argentina \\ ${ }^{3}$ Observatório Nacional, Rua General José Cristino 77, 20921-400, Rio de Janeiro, RJ, Brazil \\ ${ }^{4}$ University of Naples Federico II Physics Department "Ettore Pancini" \\ Monte Sant'Angelo Campus Via Cinthia 21, I-80126 Naples, Italy \\ ${ }^{5}$ Istituto Nazionale di Fisica Nucleare (INFN), Sez. Napoli, \\ Monte Sant'Angelo Campus Via Cinthia 9, I-80126 Naples, Italy and \\ ${ }^{6}$ Instituto de Ciencias Nucleares, Universidad Nacional Autónoma de México, A.P. 70-543, México D.F. 04510, México.
}

(Dated: Dec 2018)

The hypothesis of the self-induced collapse of the inflaton wave function was introduced as a candidate for the physical process responsible for the emergence of inhomogeneity and anisotropy at all scales. In particular, we consider different proposal for the precise form of the dynamics of the inflaton wave function: i) the GRW-type collapse schemes proposals based on spontaneous individual collapses which generate non-vanishing expectation values of various physical quantities taken as ansatz modifications of the standard inflationary scenario; ii) the proposal based on a Continuous Spontaneous Localization (CSL) type modification of the Schrödinger evolution of the inflaton wave function, based on a natural choice of collapse operator. We perform a systematic analysis within the semi-classical gravity approximation, of the standing of those models considering a full quasi-de sitter expansion scenario. We note that the predictions for the Cosmic Microwave Background (CMB) temperature and polarization spectrum differ slightly from those of the standard cosmological model. We also analyse these proposals with a Bayesian model comparison using recent $\mathrm{CMB}$ and Baryonic Acoustic Oscillations (BAO) data. Our results show a moderate preference of the joint $\mathrm{CMB}$ and $\mathrm{BAO}$ data for one of the studied collapse schemes model over the $\Lambda \mathrm{CDM}$ one, while there is no preference when only $\mathrm{CMB}$ data are considered. Additionally, analysis using CMB data provide the same Bayesian evidence for both the CSL and standard models, i.e. the data have not preference between the simplicity of the LCDM model and the complexity of the collapse scenario.

PACS numbers: $98.80 . \mathrm{Cq}, 98.70 . \mathrm{Vc}$

Keywords: cosmological parameters from $\mathrm{CMB}$, physics of the early universe, inflation

\footnotetext{
a E-mail: mpp@fcaglp.unlp.edu.ar
} 


\section{INTRODUCTION}

The assumption of an inflationary period at the very early stages of the universe's history is usually considered part of the standard cosmological model [1-4], and the physics of such period is viewed as providing an account for the observed cosmic structure [5-10]. According to this picture, during the inflationary era, the evolution of the universe is described by a Friedmann-Robertson-Walker (FRW) background cosmology with an accelerated expansion. In the simplest inflationary model, the expansion is driven by the potential of a single scalar field: the inflaton. Additionally, the quantum fluctuations of the inflaton are characterized by a simple vacuum state that is exactly symmetric, being the symmetry the homogeneity and isotropy of the quantum state. However, when considering the standard inflationary scenario more carefully an important issue arises, namely, the transition from a perfect symmetric state in the early universe to the present non-symmetric state of the current universe, which cannot be attributed to the quantum unitary evolution. This shortcoming of the inflationary scenario has been extensively discussed [11-13] and a proposal to deal with has been developed [11 20]. The most important ingredient of this proposal is to introduce the self-induced collapse hypothesis: an internally induced collapse of the inflaton wave function as the physical mechanism responsible for the emergence of inhomogeneities and anisotropies at each particular length scale. It must be emphasized that we are not calling into question the standard inflation/ $\Lambda$ CDM paradigm. Our proposal simply incorporates to the inflationary model a physical process capable of turning the homogeneity and isotropy of the vacuum state into actual inhomogeneities and anisotropies.

Our formulation of the collapse proposal assumes that at the early inflationary stage during the cosmic evolution, there was a spontaneous "jump", or a continuous series of infinitesimal "jumps", of the quantum state associated to a particular mode of the quantum field. That is very similar to what is usually taken to characterize the measurement process of Quantum Mechanics, which results in the quantum mechanical collapse of the wave function. However, in this approach, there is no external measuring device or observer that is responsible for triggering such collapse. The question one faces then is to explain the physical mechanism responsible for such spontaneous collapse. Various authors have proposed that the collapse of the wave function is a physical process induced by unknown aspects of quantum gravity $21-24]$. On the other hand, various proposals based on an objective dynamical reduction of the wave function have been developed in different contexts than the cosmological one 24 27. The aim of those proposals is to provide a solution to the quantum measurement problem, which in the particular case of cosmology is exacerbated by the absence of a well defined notion of observers, measurement devices, that might play a special role in the early universe.

It is also important to mention that the conceptual issue we are discussing is sometimes referred in the literature as the quantum-to-classical transition of the primordial perturbations [28 33] ${ }^{1}$. We note in this regard, some authors argue that decoherence [28, 30, 31, 33, 36] and/or the squeezing nature of the evolved vacuum state of the inflaton [29, 30, 37. provides a complete resolution of the problem. Nevertheless, as discussed in detail in [12, we do not endorse to such claims mainly because the squeezed nature of a quantum state can not be taken as an indication that the system has become classical, nor that the symmetries of the quantum state have disappeared. In fact, one can always find a new set of operators in which the evolved (squeezed) state will look like a "standard vacuum." For instance, if we consider the simple quantum harmonic oscillator, we can write the usual creator and annihilation operators as $\hat{a}=(1 / \sqrt{2})\left(e^{s_{0}} \hat{x}+i e^{-s_{0}} \hat{p}\right), \hat{a}^{\dagger}=(1 / \sqrt{2})\left(e^{s_{0}} \hat{x}-i e^{-s_{0}} \hat{p}\right)$ with $e^{s_{0}}=\sqrt{m \omega}$ and usual commutator relations. We can now define (without changing the system or its Hamiltonian) for arbitrary values of $s$, new operators $\hat{a}_{s}=(1 / \sqrt{2})\left(e^{s} \hat{x}+i e^{-s} \hat{p}\right), \hat{a}_{s}^{\dagger}=(1 / \sqrt{2})\left(e^{s} \hat{x}-i e^{-s} \hat{p}\right)$ which are related to the original creation and annihilation operators through a "Lorentzian rotation" and obey $\left[\hat{a}_{s}, \hat{a}_{s}^{\dagger}\right]=1$. However following the reasoning of e.g. [33] we would have that in the limit when $s \rightarrow \infty$, the fact that $\hat{x}$ and $\hat{p}$ do not commute becomes irrelevant and therefore we are in an essentially classical situation, where for each value of $\hat{a}_{s}$ there is a corresponding value of $\hat{a}_{s}^{\dagger}$. The former argument would imply that, through the simple act of choosing to express things in terms of suitable variables, we can change the nature of a purely quantum mechanical system into something which is essentially classical. The direct connection with the inflationary scenario is evidenced when nothing that the former construction is just what is used to define squeezed states. Additionally, that construction is precisely what characterizes the Bogoliubov connection between the creation annihilation operators that can be taken as natural (in the sense of leading to a simple form for the instantaneous Hamiltonian) in the early times and the corresponding ones for late times. Another approach to address the so called classicalization of the primordial perturbations, point to the Everett "many-worlds" interpretation of quantum mechanics [38 40]. However, it has been shown that none of these approaches (decoherence, squeezing of the vacuum state, many-worlds, etc.) can offer a completely satisfactory solution to the problem at hand (see

\footnotetext{
1 In fact, we find that characterizing the problem as the "quantum-to-classical" transition is somewhat misleading. Our posture is that there are no classical or quantum regimes. The fundamental description is always quantum mechanical. However, in some physical systems, there exist certain conditions that allow us to describe specific quantities, to a sufficient accuracy, by their expectation values which are then identified with their classical counterparts.
} 
refs. [11 13, 15, 17] for the conceptual and formal details of this issue). Other attempts based on non-local hidden variable theories have been considered [41, 42, but it must be noted that, just as the present one, those approaches go beyond standard quantum theory.

As we have already mentioned, once we assume the self-induced collapse of the wave function, the following task is to precisely describe the dynamics of such a process. For this purpose, there are two approaches that have been developed recently: i) a purely phenomenological approach, which is described by a general parametrization of the quantum state after collapse; we will refer to this as collapse schemes approach [14, 18, 19, 43] ii) the proposal based on the use of an adaptation to cosmology of the Continuous Spontaneous Localization (CSL) model [25 27] where a modification of the Schrödinger equation leads naturally to the eventual collapse of the inflaton wave function [20].

In the collapse schemes approach, we characterize the post-collapse state by the quantum expectation values of the field and its conjugated momentum. As a consequence of the collapse, those expectation values change from being zero, when evaluated in the vacuum state, to having non-vanishing value in the post-collapse state. Each collapse scheme leads to a particular pattern for the post-collapse expectation values, leaving an imprint in the primordial power spectrum. As a consequence the predictions for the Cosmic Microwave Background (CMB) spectrum changes with respect to the standard inflationary models one. Therefore, it can be used to constrain these models with recent observational data. In previous works, the so called independent, Newtonian and Wigner schemes have been carefully analyzed [14, 18, 19, 43]. In all cases, it has been obtained for the primordial power spectrum of scalar perturbations, an expression of the form $P(k)=A_{s} k^{n_{s}-1} Q(k)$ where $Q(k)$ is a function characterizing the details of the specific hypothesis regarding the collapse, and which, in particular takes different forms for each collapse scheme. It has been shown that for those collapse schemes, if the conformal time of collapse of each mode of the field is given by $\eta_{\vec{k}}^{c}=\mathcal{A} / k$ with $\mathcal{A}$ being a constant, then the standard prediction for primordial power spectrum of the standard inflationary accounts is recovered. Furthermore, two deviations from the aforementioned parametrization of the time of collapse have been proposed: i) $\eta_{\vec{k}}^{c}=\mathcal{A} / k+\mathcal{B} / k^{2}$ and ii) $\eta_{\vec{k}}^{c}=\mathcal{A} / k+\mathcal{B}$ with $\mathcal{B}$ a constant. Both type of deviations lead to modifications in the temperature and polarization power spectrum: for models based on i) the modifications are most pronounced at large angular scales (i.e. lowest multipoles), while the effect for the models based on ii) can be seen mainly in the smallest angular scales.

Comparison with recent CMB data, namely from the Planck collaboration (2015), has been performed for the case i) of the collapse time. In particular, we have shown that the Wigner scheme scheme provides the same Bayesian evidence as the minimal standard cosmological model $(\Lambda \mathrm{CDM}){ }^{2}$ while the Newtonian scheme is weakly disfavored with respect to the standard cosmology. On the other hand, comparison with data from the WMAP collaboration and Sloan Digital Sky Survey with the latter choice for the conformal time, has been performed in ref. [18. However, in the parametrization corresponding to case (ii), the underlying theoretical model was based on an almost exact de Sitter background $(\dot{H}=$ constant $\neq 0)$, which resulted in a theoretical prediction for the power spectrum with $n_{s}=1$. A more realistic theoretical analysis was carried out in ref. [19], where a quasi-de Sitter background was considered. In the present work, we compare the predictions of the Newton and Wigner collapse schemes for the second choice of the conformal time of collapse (ii) [19], with recent CMB data from the Planck collaboration (2015) and Baryonic Acoustic Oscillations (BAO) data.

The application of the CSL collapse model, to inflation was first analyzed in the context of semiclassical gravity with an (almost) exact de Sitter background in Ref. [20]. Furthermore the use of CSL model in the context of inflation as treated instead in terms of the Mukhanov-Sasaki variable (which involves the quantization of both the inflaton filed and the metric perturbations) has been considered in [44 50] (see also [51]). In this work, we extend the analysis within the semiclassical gravity framework while considering a quasi-de Sitter background metric. Moreover, we perform a statistical analysis to compare the predictions obtained using the CSL approach, with recent CMB data.

For all the models analyzed in this paper, we have performed a Bayesian statistical analysis and a Bayesian model comparison in order to determine, irrespective of all conceptual issues, whether the data support such a scenario when compared to the standard one based on a minimal $\Lambda \mathrm{CDM}$ model.

The paper is organized as follows: In section II] we briefly review the collapse hypothesis within the semiclassical gravity approximation and summarize both collapse mechanisms analyzed in this paper: the collapse scheme approach and the one based on the adaptation to the cosmological setting of the CSL model. In section III] we review the expressions for the primordial power spectrum calculated in ref. [19] for the collapse schemes. Furthermore, we calculate the primordial power spectrum for the CSL model in a quasi-de Sitter background for the first time. In addition, we analyze the effect of the proposed treatments on the CMB temperature angular spectrum and describe the results in terms of an appropriate parametrization. Afterwards, in section IV, we introduce the computational and statistical tools, and the data set used in our analysis. In section $\mathrm{V}$, we present the results of our analysis and

\footnotetext{
${ }^{2}$ By standard cosmological model $(\Lambda \mathrm{CDM})$ we understand a specific choice of the cosmological parameters plus the standard inflationary model, in contrast with the collapse models, where the collapse hypothesis is assumed for inflation and the cosmological parameters remain unchanged
} 
the constraints on the cosmological and collapse parameters. Finally, in section VI] we summarize the main results of the paper and present our conclusions.

\section{THE MODEL}

In this section, we briefly review the key aspects of inflationary models with a self-induced collapse of the inflaton wave function. For a detailed description of this framework, we refer the reader to refs. [11, 14, 18, 19]. Regarding notation and conventions, we will work with signature $(-,+,+,+)$ for the metric; primes over functions will denote derivatives with respect to the conformal time $\eta$, and we will use units where $c=\hbar=1$ but keep the gravitational constant $G$.

At this point it is worthwhile clarifying the general ideology behind the manner we investigate the interface between gravitation and quantum. Most modern research programs concerned about this question start by postulate the full quantum gravity theory inspired by lines of thought that lead to conjectures about what ought to be taken as fundamental language to deal with the issue, mathematical elegance and so forth, (for instance LQG or String theory or Causal Sets approach) and then the program seeks to make connection with theories whose validity in the appropriate regime is taken as well established (e.g. General Relativity or Quantum Field Theory) or even sometimes the quest is directly to seek connections with the empirical accessible world. These approaches often face the questions of how to connect with observations, and substantial amount of work, some of it involving "reasonable guesses" (but guesses nonetheless), to even reach that point. That is, what we call the bottom-up approach to physics. We approach the issue in the opposite direction, using what we call the top-down approach: This is based on being agnostic regarding the nature of the fundamental theory, and instead considering extrapolation of rather well stablished theories (General Relativity together with Quantum Field Theory in curved space-time) to regimes where such inquires are expected to face very delicate issues. We then try to find simple manners to bridge these difficulties and then study whether or not the results are reasonable (i.e do they agree with observations? or do they fit together with other clues about the regimes of interest?). This is not to be construed as a criticism to the standard approaches. We in fact think the two are reasonable paths of inquire, but the point is that we must recognize that each one of those faces the most difficult problems are at different stages of the program.

As in standard slow-roll inflationary models, we consider the action of a single scalar field, minimally coupled to gravity, with an appropriate potential:

$$
S\left[\phi, g_{a b}\right]=\int d^{4} x \sqrt{-g}\left[\frac{1}{16 \pi G} R[g]-\frac{1}{2} \nabla_{a} \phi \nabla_{b} \phi g^{a b}-V[\phi]\right] .
$$

The background metric is described by a FRW spacetime. In conformal coordinates, the components of the background metric are $g_{\mu \nu}^{(0)}=a(\eta) \eta_{\mu \nu}$, with $\eta$ the conformal cosmological time and $\eta_{\mu \nu}$ the components of the Minkowskian metric, and $a(\eta)$ is the scale factor. During slow-roll inflation, the scale factor can be approximated by $a(\eta) \simeq-1 /\left[H \eta\left(1-\epsilon_{1}\right)\right]$, with $H$ the Hubble factor, which during inflation is approximately constant. The Hubble slow-roll parameters, are defined as $\epsilon_{1} \equiv 1-\mathcal{H}^{\prime} / \mathcal{H}^{2}, \epsilon_{2} \equiv \epsilon_{1}^{\prime} / \mathcal{H} \epsilon_{1}$, and both are very small $\epsilon_{1}, \epsilon_{2} \ll 1$. Here $\mathcal{H} \equiv a H$ is the conformal Hubble factor. Furthermore, in context of the slow-roll approximation the slow-roll parameters are related to the inflaton potential through,

$$
\begin{gathered}
\epsilon_{1} \simeq \frac{M_{P}^{2}}{2}\left(\frac{\partial_{\phi} V}{V}\right)^{2}, \\
\epsilon_{2} \simeq 2 M_{P}^{2}\left[\left(\frac{\partial_{\phi} V}{V}\right)^{2}-\left(\frac{\partial_{\phi \phi} V}{V}\right)\right] .
\end{gathered}
$$

Within the slow-roll approximation, the equation of motion for the background field is $3 \mathcal{H} \phi_{0}^{\prime}=-a^{2} \partial_{\phi} V$.

The standard procedure is to split the scalar field and the metric into background plus perturbations, i.e. $g_{a b}=$ $g_{a b}^{(0)}+\delta g_{a b}$ and $\phi(\vec{x}, \eta)=\phi_{0}(\eta)+\delta \phi(\vec{x}, \eta)$. At leading order in the scalar perturbations of the background metric 3

\footnotetext{
3 In recent works [52 54, we have focused on the tensor perturbations of the metric in the context of the semi-classical approach used in this paper. The results of those works indicate that the corresponding tensor modes are strongly suppressed. Therefore, in this paper, we only consider scalar perturbations of the metric.
} 
assuming no anisotropic stress, and working in the longitudinal gauge, imply that the line element associated to the scalar metric perturbations is

$$
d s^{2}=a^{2}(\eta)\left[-(1-2 \Psi) d \eta^{2}+(1-2 \Psi) \delta_{i j} d x^{i} d x^{j}\right] .
$$

Before addressing the modified quantum dynamics and its impact in the treatment of the infationary cosmology, we present the framework that underlies our characterization of space-time metric and that of the inflaton field $4^{4}$ This framework is based on the semiclassical gravity approach, in which the matter fields are treated quantum mechanically while the gravity is treated in classical terms 5 Note that this is a distinct view from the standard approach in which the perturbations of both the metric and the matter fields are treated in quantum mechanical terms. The framework we employ is thus based on Einstein's semiclassical equations,

$$
G_{a b}=8 \pi G\left\langle\hat{T}_{a b}\right\rangle .
$$

We must mention the fact that direct calculations indicate the quantum uncertainties in $\hat{T}_{a b}$ in this situation are in principle very large [55] (in fact strictly speaking and when considered at a given spacetime point these would be infinite), and that might be taken as casting doubts about the validity not only of semiclassical gravity but also of any kind of perturbative approach underlying all treatments of cosmological perturbations. Nevertheless, the fact that one obtains reasonable results, indicates the problem is not insurmountable, and that some cutoff mechanism must be at play ${ }^{6}$ It should be noted however that in the assessment of these issues one should be careful to compare the uncertainties in the energy momentum tensor with the expectation value of a full energy momentum tensor (a quantity that is extremely large during inflation) and not just that of the space dependent perturbations. The problem however clearly deserves further study.

In our approach the initial state of the quantum field is taken to be the same as the standard one, namely the BunchDavies vacuum. Nevertheless, the self-induced collapse will spontaneously change this initial state into a final one that does not need to share the symmetries of the Bunch-Davies vacuum. Henceforth, after the collapse $\left\langle\hat{T}_{a b}\right\rangle$ will not have the symmetries of the initial state, and this will led through Einstein semiclassical equation, to a geometry, that. generically, will no longer be homogeneous and isotropic. In particular, focusing on the metric scalar perturbation $\Psi$ Einstein's semiclassical equations in Fourier space, at first order in the perturbation theory, led to:

$$
\Psi_{\vec{k}}(\eta)=\sqrt{\frac{\epsilon_{1}}{2}} \frac{H}{M_{P} k^{2}} a\left\langle\hat{\delta}_{\vec{k}}^{\prime}(\eta)\right\rangle,
$$

where $M_{P}^{2} \equiv 1 / 8 \pi G$ the reduced Planck mass. Considering an homogeneous and isotropic vacuum state for the field would lead to $\left\langle 0\left|\hat{\delta \phi}_{\vec{k}}^{\prime}\right| 0\right\rangle=0$. It follows from eq. (5) that in the vacuum state $\Psi=0$ and consequently the spacetime is perfectly homogeneous and isotropic. It is only after after the self-induced collapse of the wave function, associated to each mode of the inflaton, that $\left\langle\hat{\delta \phi}_{\vec{k}}^{\prime}\right\rangle \neq 0$, giving rise to the emergence of the primordial curvature perturbations.

Note that, in the standard treatment, there is no analogous expression to Eq. (5). In fact, the usual treatment is based on the quantization of both $\Psi$ and $\delta \phi$, which then are linearly combined in what is called the Mukhanov-Sasaki variable $v$ [56]. Essentially, the treatment starts with the action at second order in perturbations, in a quasi-de Sitter spacetime background, expressed in terms of the variable $v$. Such an action is then expanded in Fourier modes, which takes the form (for each mode) of a harmonic oscillator with a "time-dependent mass". This is followed by the canonical quantization of $v$. In the comoving gauge the variable $v$ and the curvature perturbation $\mathcal{R}$ (i.e the intrinsic spatial curvature on hypersurfaces on constant conformal time for a flat universe) are related by $\mathcal{R}=v / z$, with $z \equiv a \sqrt{2 \epsilon_{1}} M_{P}$. Hence, a quantization of $v$ implies a quantization of $\mathcal{R}$. Additionally, the traditional approach assumes that when the proper wavelength of the mode becomes larger than the Hubble radius, a certain quantum to classical transition takes place, which might be expressed as $\hat{\mathcal{R}}_{\vec{k}} \rightarrow \mathcal{R}_{\vec{k}}$ (the justification for assuming such transition usually relies on arguments based on decoherence, the evolution of the vacuum state into a squeezed state, etc.). That is the quantum operator $\hat{\mathcal{R}}_{\vec{k}}$ is now taken as a classical stochastic field $\mathcal{R}_{\vec{k}}=A(k) e^{i \theta_{\vec{k}}}$. The quantity $\theta_{\vec{k}}$ is a random phase, and $A(k)$ is a Gaussian random variable with zero mean and variance directly identified with the quantum uncertainty $\left\langle 0\left|\hat{\mathcal{R}}_{\vec{k}}^{2}\right| 0\right\rangle$. That is, the focus of the standard approach is the two-point quantum correlation function $\left\langle 0\left|\hat{\mathcal{R}}_{\vec{k}} \hat{\mathcal{R}}_{\vec{k}^{\prime}}^{\dagger}\right| 0\right\rangle \equiv P(k) \delta\left(\vec{k}-\vec{k}^{\prime}\right)$ from where the power spectrum $P(k)$ is extracted.

\footnotetext{
${ }_{5}^{4}$ For a further detailed presentation and motivation for our approach see ref. [11, 12].

5 The point of view accepts that spacetime is quantum mechanical at the fundamental level, but considers that by the time that a metric characterization is meaningful, one is already well within the classical realm as far as the gravitational degrees of freedom are concerned. This view is quite natural once one considers, say the problem of time in canonical quantum gravity, and the regimes in which a notion of time might effectively emerge.

${ }^{6}$ For instance in [53, 54] it was argued that when considering the spectrum at the end of inflation, it was natural to take a cutoff scale to be given by the last scale that exits the horizon during inflation. On the other hand, when one is interested in comparing the theoretical predictions with the data from the CMB, one must take into account plasma damping effects and thus introduce a cutoff scale corresponding to the scale of diffusion or Silk damping
} 
In the next subsection, we will focus on the strictly semiclassical approach 7 based on quantum treatment of the matter fields and describe the modified dynamics corresponding to the self-induced collapse. There are two main approaches that will be considered in this paper: i) the one in which no particular collapse mechanism is considered, and the collapse process is simply characterized in a phenomenologically inspired scheme [14, 18, 19; ii) a second approach where a modification of the Schrödinger equation of the CSL type leads naturally to the eventual collapse of the wave function [20. As mentioned in the introduction, we refer to the first as the collapse schemes approach, and the second one as the continuous spontaneous localization (CSL) inflationary approach.

\section{A. Quantum treatment and Collapse schemes}

The staring point of the treatment is the quantum theory of $\delta \phi(\vec{x}, \eta)$ in a curved background described by a quasi-de Sitter space-time [14, 18, 19]. Moreover, it is convenient to work with the rescaled field variable $y=a \delta \phi$. Both the field $y$ and the canonical conjugated momentum $\pi \equiv \partial \delta \mathcal{L}^{(2)} / \partial y^{\prime}=y^{\prime}-\left(a^{\prime} / a\right) y=a \delta \phi^{\prime}$ are promoted to quantum operators so that they satisfy the following equal time commutator relations: $\left[\hat{y}(\vec{x}, \eta), \hat{\pi}\left(\vec{x}^{\prime}, \eta\right)\right]=i \delta\left(\vec{x}-\vec{x}^{\prime}\right)$ and $\left[\hat{y}(\vec{x}, \eta), \hat{y}\left(\vec{x}^{\prime}, \eta\right)\right]=\left[\hat{\pi}(\vec{x}, \eta), \hat{\pi}\left(\vec{x}^{\prime}, \eta\right)\right]=0$. Next, we can expand the field operator in Fourier modes,

$$
\hat{y}(\eta, \vec{x})=\frac{1}{L^{3}} \sum_{\vec{k}} \hat{y}_{\vec{k}}(\eta) e^{i \vec{k} \cdot \vec{x}},
$$

with an analogous expression for $\hat{\pi}(\eta, \vec{x})$. Note that the sum is over the wave vectors $\vec{k}$ satisfying $k_{i} L=2 \pi n_{i}$ for $i=1,2,3$ with $n_{i}$ integer and $\hat{y}_{\vec{k}}(\eta) \equiv y_{k}(\eta) \hat{a}_{\vec{k}}+y_{k}^{*}(\eta) \hat{a}_{-\vec{k}}^{\dagger}$ and $\hat{\pi}_{\vec{k}}(\eta) \equiv g_{k}(\eta) \hat{a}_{\vec{k}}+g_{k}^{*}(\eta) \hat{a}_{-\vec{k}}^{\dagger}$, with $g_{k}(\eta)=y_{k}^{\prime}(\eta)-\mathcal{H} y_{k}(\eta)$. The equation of motion for the modes reads

$$
y_{k}^{\prime \prime}(\eta)+\left(k^{2}-\frac{2+3 \epsilon}{\eta^{2}}\right) y_{k}(\eta)=0,
$$

with $\epsilon \equiv-\epsilon_{1}+\epsilon_{2} / 2$. The selection of $y_{k}(\eta)$ reflects the choice of a vacuum state for the field. We proceed as in standard inflationary models and choose the so-called Bunch-Davies vacuum:

$$
y_{k}(\eta)=\left(\frac{-\pi \eta}{4}\right)^{1 / 2} e^{i[\nu+1 / 2](\pi / 2)} H_{\nu}^{(1)}(-k \eta)
$$

where $\nu \equiv 3 / 2+\epsilon$ and $H_{\nu}^{(1)}(-k \eta)$ is the Hankel function of the first kind of order $\nu$.

The collapse hypothesis assumes that at a certain time $\eta_{\vec{k}}^{c}$ the part of the state characterizing the mode $k$ randomly "jumps" to a new state, which is no longer homogeneous and isotropic. The collapse is considered to operate similar to an imprecise "measurement," even though there is no external observer or detector involved. Therefore, it is reasonable to consider Hermitian operators, which are susceptible of a direct measurement in ordinary quantum mechanics. Hence, we separate $\hat{y}_{\vec{k}}(\eta)$ and $\hat{\pi}_{\vec{k}}(\eta)$ into their real and imaginary parts $\hat{y}_{\vec{k}}(\eta)=\hat{y}_{\vec{k}}{ }^{R}(\eta)+i \hat{y}_{\vec{k}}{ }^{I}(\eta)$ and $\hat{\pi}_{\vec{k}}(\eta)=\hat{\pi}_{\vec{k}}^{R}(\eta)+i \hat{\pi}_{\vec{k}}^{I}(\eta)$, such that the operators $\hat{y}_{\vec{k}}^{R, I}(\eta)$ and $\hat{\pi}_{\vec{k}}^{R, I}(\eta)$ are Hermitian operators. Thus,

$$
\begin{aligned}
& \hat{y}_{\vec{k}}^{R, I}(\eta)=\sqrt{2} \operatorname{Re}\left[y_{k}(\eta) \hat{a}_{\vec{k}}^{R, I}\right], \\
& \hat{\pi}_{\vec{k}}^{R, I}(\eta)=\sqrt{2} \operatorname{Re}\left[g_{k}(\eta) \hat{a}_{\vec{k}}^{R, I}\right],
\end{aligned}
$$

where $\hat{a}_{\vec{k}}^{R} \equiv\left(\hat{a}_{\vec{k}}+\hat{a}_{-\vec{k}}\right) / \sqrt{2}, \hat{a}_{\vec{k}}^{I} \equiv-i\left(\hat{a}_{\vec{k}}-\hat{a}_{-\vec{k}}\right) / \sqrt{2}$.

The commutation relations for the $\hat{a}_{\vec{k}}^{R, I}$ are non-standard:

$$
\left[\hat{a}_{\vec{k}}^{R, I}, \hat{a}_{\vec{k}^{\prime}}^{R, I \dagger}\right]=L^{3}\left(\delta_{\vec{k}, \vec{k}^{\prime}} \pm \delta_{\vec{k},-\vec{k}^{\prime}}\right),
$$

the + and the - sign corresponds to the commutator with the $R$ and $I$ labels respectively; all other commutators vanish. It is also important to emphasize that the vacuum state defined by $\hat{a}_{\vec{k}}{ }^{R, I}|0\rangle=0$ is fully translational and rotationally invariant (see the formal proof in Appendix A of ref. [13]).

7 Such approach is sometimes considered as not viable, but as the discussion illustrated in [57-63] the arguments are not decisive. In particular, an implementation involving a self-induced collapse seems to be completely viable as far as mathematical consistency and phenomenology are concerned [64, 65, and at least when regarding these as effective theories. 
Next, we need to specify the dynamics of the expectation values $\left\langle\hat{y}_{\vec{k}}^{R, I}(\eta)\right\rangle$ and $\left\langle\hat{\pi}_{\vec{k}}^{R, I}(\eta)\right\rangle$, evaluated in the postcollapse state, which will depend on the expectation values evaluated at the time of collapse of each mode of the field $\eta_{\vec{k}}^{c}$. In the collapse schemes approach, we do not consider a specific collapse mechanism, instead we characterize the post-collapse state by the expectation value and the quantum uncertainty of the fields at the time $\eta_{\vec{k}}^{c}$. In the present work, we will consider only two possibilities for such relations. Namely, the Newtonian and Wigner collapses schemes analyzed in ref. [19]. We do not consider the independent scheme studied in the same work since it has been shown [18, 19] that when considering the collapse time $\eta_{c}^{k}=\frac{A}{k}+B$, the CMB angular spectrum, associated to that scheme, is indistinguishable from the prediction of the standard inflationary model.

\section{Newtonian collapse scheme}

This scheme is motivated by the fact that only the expectation value of the momentum operator $\hat{\pi}_{\vec{k}} \equiv a \hat{\delta}_{\vec{k}}^{\prime}$ appears as a source for the curvature perturbation $\Psi_{\vec{k}}$ in Eq. (5). Also, this view seems to be close in spirit to the ideas of Penrose 21] regarding quantum uncertainties that the gravitational potential would be inheriting from the matter fields' quantum uncertainties. Therefore, in this scheme the collapse affects only the expectation value of the conjugated momentum variable, i.e.,

$$
\left\langle\hat{y}_{\vec{k}}^{R, I}\left(\eta_{\vec{k}}^{c}\right)\right\rangle=0, \quad\left\langle\hat{\pi}_{\vec{k}}^{R, I}\left(\eta_{\vec{k}}^{c}\right)\right\rangle=x_{\vec{k}, 2}^{R, I} \sqrt{\left(\Delta \hat{\pi}_{\vec{k}}^{R, I}\left(\eta_{\vec{k}}^{c}\right)\right)_{0}^{2}}
$$

where, $x_{\vec{k}, 2}^{(R, I)}$ represents a random Gaussian variable normalized and centered at zero. The quantum uncertainties of the vacuum state at the time of collapse are [19]:

$$
\begin{aligned}
\left(\Delta \hat{y}_{\vec{k}}^{R, I}\left(\eta_{\vec{k}}^{c}\right)\right)_{0}^{2} & =\frac{L^{3} \pi\left|z_{k}\right|}{16 k}\left[J_{\nu}^{2}\left(\left|z_{k}\right|\right)+Y_{\nu}^{2}\left(\left|z_{k}\right|\right)\right], \\
\left(\Delta \hat{\pi}_{\vec{k}}^{R, I}\left(\eta_{\vec{k}}^{c}\right)\right)_{0}^{2} & =\frac{L^{3} \pi k}{16}\left[\left(\frac{-\alpha J_{\nu}\left(\left|z_{k}\right|\right)}{\sqrt{\left|z_{k}\right|}}\right.\right. \\
& \left.+\sqrt{z_{k} \mid} J_{\nu+1}\left(\left|z_{k}\right|\right)\right)^{2}+\left(\frac{-\alpha Y_{\nu}\left(\left|z_{k}\right|\right)}{\sqrt{\left|z_{k}\right|}}\right. \\
& \left.\left.+\sqrt{\left|z_{k}\right|} Y_{\nu+1}\left(\left|z_{k}\right|\right)\right)^{2}\right],
\end{aligned}
$$

where $\alpha \equiv 1 / 2+\nu, J_{\nu}$ and $Y_{\nu}$ are the Bessel functions of the first and second kind respectively; $z_{k} \equiv k \eta_{\vec{k}}^{c}$ and $\eta_{\vec{k}}^{c}$ is the time of collapse for each mode.

\section{Wigner collapse scheme}

Heissenberg's uncertainty principle indicates that quantum uncertainties of position and momentum operators are not independent. In particular, momentum and position of a quantum system cannot be determined independently and simultaneously. As we have mentioned, the self-induced collapse acts as a sort of spontaneous "measurement" (of course without relying on observers or measurements devices) of some variable involving both position and momentum. Therefore, as suggested by the uncertainty principle, the collapse might involve correlations between position and momentum. Extrapolating this idea to our situation of interest indicates that the self-induced collapse could correlate the field $\hat{y}$ and its conjugated momentum $\hat{\pi}$. One possible way to characterize the correlation is to use Wigner's distribution function. In non-relativistic quantum mechanics, Wigner's function can be considered, under certain special circumstances, as a probability distribution function for a quantum system, i.e. it allow us to visualize the momentum-position correlations and quantum interferences in "phase space". For the vacuum state of each mode of the inflaton, the corresponding Wigner's function is a bi-dimensional Gaussian. As a consequence, in this scheme we will characterize the post-collapse expectation values as:

$$
\left\langle\hat{y}_{\vec{k}}^{R, I}\left(\eta_{\vec{k}}^{c}\right)\right\rangle=x_{\vec{k}}^{R, I} \Lambda_{k}\left(\eta_{\vec{k}}^{c}\right) \cos \Theta_{k}\left(\eta_{\vec{k}}^{c}\right),
$$




$$
\left\langle\hat{\pi}_{\vec{k}}^{R, I}\left(\eta_{\vec{k}}^{c}\right)\right\rangle=x_{\vec{k}}^{R, I} k \Lambda_{k}\left(\eta_{\vec{k}}^{c}\right) \sin \Theta_{k}\left(\eta_{\vec{k}}^{c}\right),
$$

where $x_{\vec{k}}^{R, I}$ is a random variable, characterized by a Gaussian probability distribution function centered at zero with spread one. The parameter $\Lambda_{k}\left(\eta_{\vec{k}}^{c}\right)$ represents the major semi-axis of the ellipse in the $\hat{y}-\hat{\pi}$ plane where the Wigner function has $1 / 2$ of its maximum value. The other parameter $\Theta_{k}\left(\eta_{\vec{k}}^{c}\right)$ is the angle between $\Lambda_{k}\left(\eta_{\vec{k}}^{c}\right)$ and the $\hat{y}_{\vec{k}}^{R, I}$ axis. For details involving the Wigner function and the collapse scheme we refer the reader to ref. [14]. The explicit expressions for $\Lambda_{k}$ [19] and $\Theta_{k}$ are

$$
\begin{gathered}
\Lambda_{k}=(2 L)^{3 / 2} \sqrt{\frac{\pi\left|z_{k}\right|}{4 k}}\left[J_{\nu}^{2}\left(\left|z_{k}\right|\right)+Y_{\nu}^{2}\left(\left|z_{k}\right|\right)\right]^{1 / 2}\left[S\left(\left|z_{k}\right|\right)\right. \\
\left.-\sqrt{S^{2}\left(\left|z_{k}\right|\right)-\left(\frac{\pi\left|z_{k}\right|}{2}\right)^{2}\left(J_{\nu}^{2}\left(\left|z_{k}\right|\right)+Y_{\nu}^{2}\left(\left|z_{k}\right|\right)\right)^{2}}\right]^{-1 / 2} \\
\tan 2 \Theta_{k}=-\frac{\pi^{2}\left|z_{k}\right|}{4}\left[J_{\nu}^{2}\left(\left|z_{k}\right|\right)+Y_{\nu}^{2}\left(\left|z_{k}\right|\right)\right]\left[S\left(\left|z_{k}\right|\right)\right. \\
\left.-\frac{\pi\left|z_{k}\right|}{8}\left(J_{\nu}^{2}\left(\left|z_{k}\right|\right)+Y_{\nu}^{2}\left(\left|z_{k}\right|\right)\right)^{2}\right]^{-1} \\
\times\left[-2 \nu\left(J_{\nu}^{2}\left(\left|z_{k}\right|\right)+Y_{\nu}^{2}\left(\mid z_{k}\right)\right)\right. \\
+\left|z_{k}\right|\left(J_{\nu}\left(\left|z_{k}\right|\right) J_{\nu+1}\left(\left|z_{k}\right|\right)\right. \\
\left.\left.+Y_{\nu}\left(\left|z_{k}\right|\right) Y_{\nu+1}\left(\left|z_{k}\right|\right)\right)\right]
\end{gathered}
$$

where

$$
\begin{aligned}
S\left(\left|z_{k}\right|\right) & \equiv 1+\frac{\pi^{2}}{16}\left\{\left|z_{k}\right|^{2}\left(J_{\nu}^{2}\left(\left|z_{k}\right|\right)+Y_{\nu}^{2}\left(\left|z_{k}\right|\right)\right)^{2}\right. \\
& +4\left[J_{\nu}^{2}\left(\left|z_{k}\right|\right)+Y_{\nu}^{2}\left(\left|z_{k}\right|\right)-\left|z_{k}\right|\left(J_{\nu}\left(\left|z_{k}\right|\right)\right.\right. \\
& \left.\left.\left.\times J_{\nu+1}\left(\left|z_{k}\right|\right)+Y_{\nu}\left(\left|z_{k}\right|\right) Y_{\nu+1}\left(\left|z_{k}\right|\right)\right)\right]^{2}\right\} .
\end{aligned}
$$

\section{B. CSL inflationary approach}

The implementation of the CSL model into the slow-roll inflationary model, using the semiclassical gravity framework, has been analyzed originally in ref. [20]. Here, we provide the main features of the CSL inflationary model generalized into a quasi-de Sitter spacetime background.

The CSL model is based on a modification of the Schrödinger equation. This alteration induces a collapse of the wave function towards one of the possible eigenstates of an operator called the collapse operator with certain rate. The objective reduction process is due to the interaction of the system with a background noise, which is a continuous-time stochastic process of the Wiener kind (see [26, 27] for a throughly review). We will be more precise in the following.

In order to apply the CSL model to the inflationary setting, we will follow the approach first introduced in [20]. That work relies on a version of the CSL model in which the nonlinear aspects of the CSL model are shifted to the probability law. That is, the evolution law is linear just as the Schrödinger equation, but then, the law of probability for the realization of a specific random function, becomes dependent of the state that results from such evolution. Specifically, the theory can be characterized in terms of two equations: The first is a modified Schrödinger equation, whose solution is

$$
|\psi, t\rangle=\mathcal{T} e^{-\int_{0}^{t} d t^{\prime}\left[i \hat{H}+\frac{1}{4 \lambda}\left[w\left(t^{\prime}\right)-2 \lambda_{0} \hat{A}\right]^{2}\right]}|\psi, 0\rangle .
$$

$\mathcal{T}$ is the time-ordering operator, $w(t)$ characterizes the stochastic process, i.e. is a random classical function of time, of white noise type. The modification of Schrödinger's equation given by the CSL model induces the collapse of 
the wave function towards one of the possible eigenstates of $\hat{A}$, that is, the operator $\hat{A}$ is the collapse operator. In laboratory situations, the collapse operator is usually chosen to be the position operator [27]. The parameter $\lambda_{0}$ is the universal CSL parameter that serves to set the strength of the collapse. The value of $\lambda_{0}$ characterizes the rate at which the wave function increases its "localizations" in the eigen-basis of the collapse operator.

The probability for the $w(t)$ is given by the second equation, the Probability Rule

$$
P D w(t) \equiv\langle\psi, t \mid \psi, t\rangle \prod_{t_{i}=0}^{t} \frac{d w\left(t_{i}\right)}{\sqrt{2 \pi \lambda / d t}} .
$$

In Ref. [20] it is shown that with the appropriate selection of the field collapse operators and using the corresponding CSL evolution law one obtains collapse in the relevant operators corresponding to the Fourier components of the field and the momentum conjugate of the field. This bypasses any concerns regarding possible mode mixing at the first order in perturbation theory (at higher order there is mode mixing even in the traditional treatments) 8

Given that the CSL model modifies the Schrödinger equation, it is convenient to describe the quantum theory of the inflaton in the Schrödinger picture, where the relevant objects are the wave function and the Hamiltonian.

The Hamiltonian characterizing the inhomogeneous sector of the inflaton is $H=(1 / 2) \int d^{3} k\left(H_{\vec{k}}^{\mathrm{R}}+H_{\vec{k}}^{\mathrm{I}}\right)$ with

$$
H_{\vec{k}}^{\mathrm{R}, \mathrm{I}}=\pi_{\vec{k}}^{\mathrm{R}, \mathrm{I}} \pi_{\vec{k}}^{* \mathrm{R}, \mathrm{I}}+k^{2} y_{\vec{k}}^{\mathrm{R}, \mathrm{I}} y_{\vec{k}}^{* \mathrm{R}, \mathrm{I}}-\frac{\left(1-\epsilon_{1}+\epsilon_{2} / 2\right)}{\eta}\left(y_{\vec{k}}^{\mathrm{R}, \mathrm{I}} \pi_{\vec{k}}^{* \mathrm{R}, \mathrm{I}}+y_{\vec{k}}^{* \mathrm{R}, \mathrm{I}} \pi_{\vec{k}}^{\mathrm{R}, \mathrm{I}}\right)
$$

where $y_{\vec{k}}=a \delta \phi_{\vec{k}}$ and $\pi_{\vec{k}} \equiv y_{\vec{k}}^{\prime}-\mathcal{H} y_{\vec{k}}$. The indexes R,I denote the real and imaginary parts of $y_{\vec{k}}$ and $\pi_{\vec{k}}$. We remind the reader that $\epsilon_{1}$ and $\epsilon_{2}$ are the Hubble slow roll parameters defined at the beginning of this section. We now promote $y_{\vec{k}}$ and $\pi_{\vec{k}}$ to quantum operators, by imposing canonical commutations relations $\left[\hat{y}_{\vec{k}}^{\mathrm{R}, \mathrm{I}}, \hat{\pi}_{\vec{k}}^{\mathrm{R}, \mathrm{I}}\right]=i \delta\left(\vec{k}-\vec{k}^{\prime}\right)$.

In order to apply the CSL theory to the situation at hand we need to make an educated guess regarding the collapse operator $\hat{A}$ that should drive the modified dynamics in this case. As we explained in the case of nonrelativistic quantum mechanics the operator $\hat{A}$ is taken as a smeared position operator, which could be associated with a sort of mass density (specially if the collapse parameter is proportional to the particle's mass as suggested in a previous work[27]). One might interpret that view as indicating that the collapse is tied with some aspect of the quantum matter that "gravitates" (i.e. that would characterize the interaction between gravitation and matter degrees of freedom). Thus, extrapolating that idea to the situation at hand, we can guess that we should look at the quantity appearing in the relevant component of Einstein's semiclassical equation as a candidate for the collapse operator. Considering now the form of the relevant component of such equation given by Eq. 5 this line of thought takes us to consider the momentum conjugate to the field as a rather natural candidate. For reasons mentioned at the beginning of this subsection, one may apply the CSL reduction mechanism on each mode of the field independently. That is, we assume that the momentum operator $\hat{\pi}_{\vec{k}}^{\mathrm{R}, \mathrm{I}}$ in each mode acts as the collapse operator for that mode. However, as in any such situation, the suitability of an educated guess must be decided by the long term empirical success or failure of the emerging predictions. Therefore, the evolution of the state vector characterizing the quantum field as given by the CSL theory is:

$$
\left|\Phi_{\vec{k}}^{\mathrm{R}, \mathrm{I}}, \eta\right\rangle=\hat{T} \exp \left\{-\int_{\tau}^{\eta} d \eta^{\prime}\left[i \hat{H}_{\vec{k}}^{\mathrm{R}, \mathrm{I}}+\frac{1}{4 \lambda_{k}}\left(\mathcal{W}\left(\vec{k}, \eta^{\prime}\right)-2 \lambda_{k} \hat{\pi}_{\vec{k}}^{\mathrm{R}, \mathrm{I}}\right)^{2}\right]\right\}\left|\Phi_{\vec{k}}^{\mathrm{R}, \mathrm{I}}, \tau\right\rangle,
$$

$\hat{T}$ is the time-ordering operator, and $\tau$ denotes the conformal time at the beginning of inflation. In addition, we have generalized the white noise $w(t)$ appearing in Eq. (18) into a stochastic field $\mathcal{W}$ which depends on $\vec{k}$ and the conformal time. That is, since we are applying the CSL collapse dynamics to each mode of the field, it is natural to introduce a stochastic function for each independent degree of freedom. Henceforth, the stochastic field $\mathcal{W}(\vec{k}, \eta)$ might be regarded as a Fourier transform on a stochastic spacetime field $\mathcal{W}(\vec{x}, \eta)$.

Given that we take the momentum operator $\hat{\pi}_{\vec{k}}^{\mathrm{R}, \mathrm{I}}$ to act as the collapse operator, it is convenient to work with the wave function in the momentum representation. We denote by $\Phi[\pi]$ the wave function characterizing the quantum state of the field. In Fourier space, the wave function can be factorized into mode components $\Phi[\pi]=\Pi_{\vec{k}} \Phi_{\vec{k}}^{\mathrm{R}}\left[\pi_{\vec{k}}^{\mathrm{R}}\right] \times \Phi_{\vec{k}}^{\mathrm{I}}\left[\pi_{\vec{k}}^{\mathrm{I}}\right]$.

It is known that the ground state of the Hamiltonian (20) characterized by a wave functional in the momentum representation $\Phi_{0}^{\mathrm{R}, \mathrm{I}}\left(\pi_{\vec{k}}^{\mathrm{R}, \mathrm{I}}\right)$ is a Gaussian. Additionally, the Hamiltonian 20) and the CSL evolution equation 21) are

\footnotetext{
8 We also acknowledge at this point that there is no complete version of the CSL theory that is applicable in all situations, ranging from laboratory ones to the ones involving cosmology and black hole space-times. Nevertheless, we adopt the point of view that proposing educated guesses, in combination with phenomenological models applicable to particular situations, allow us to progress in our program. We think this is analogous to the path that took physics down the road that ended with the standard model of particle physics, namely trial and error focusing first on rather specific situations, and then looking for ways to generalize, based on what was found to work in each case.
} 
quadratic in both $\hat{\pi}_{\vec{k}}^{\mathrm{R}, \mathrm{I}}$ and $\hat{y}_{\vec{k}}^{\mathrm{R}, \mathrm{I}}$; consequently, the form of the wave function at any time in the momentum basis is 20]:

$$
\Phi^{\mathrm{R}, \mathrm{I}}\left(\eta, \pi_{\vec{k}}^{\mathrm{R}, \mathrm{I}}\right)=\exp \left[-A_{k}(\eta)\left(\pi_{\vec{k}}^{\mathrm{R}, \mathrm{I}}\right)^{2}+B_{k}^{\mathrm{R}, \mathrm{I}}(\eta) \pi_{\vec{k}}^{\mathrm{R}, \mathrm{I}}+C_{k}^{\mathrm{R}, \mathrm{I}}(\eta)\right] .
$$

The evolution equation (21) when applied to the wave functional 222 , results in a set of dynamical equations for the objects $A_{k}(\eta), B_{k}^{\mathrm{R}, \mathrm{I}}(\eta)$, and $C_{k}^{\mathrm{R}, \mathrm{I}}(\eta)$. The initial conditions are set by the initial state of the field, i.e. the Bunch-Davies vacuum. That is, the initial conditions are $A_{k}(\tau)=1 / 2 k, B_{k}^{\mathrm{R}, \mathrm{I}}(\tau)=0$, and $C_{k}^{\mathrm{R}, \mathrm{I}}(\tau)=0$. As a matter of fact, we are only interested in the equation of motion for $A_{k}(\eta)$ since this quantity is directly related to the primordial spectrum. The analysis of [20] in fact indicates that,

$$
A_{k}^{\prime}=\frac{i}{2}+\lambda_{k}-2 A_{k} \frac{\left(1-\epsilon_{1}+\epsilon_{2} / 2\right)}{\eta}-2 i k^{2} A_{k}^{2} .
$$

The solution of the latter equation is

$$
A_{k}(\eta)=\frac{q}{2 i k^{2}}\left[\frac{J_{\mu+1}(-q \eta)+e^{-i \pi \mu} J_{-\mu-1}(-q \eta)}{J_{\mu}(-q \eta)-e^{-i \pi \mu} J_{-\mu}(-q \eta)}\right]
$$

with $q^{2} \equiv k^{2}\left(1-2 i \lambda_{k}\right)$ and $\mu \equiv 1 / 2-\epsilon_{1}+\epsilon_{2} / 2$

\section{PRIMORDIAL POWER SPECTRUM FOR THE COLLAPSE MODELS}

In this section, we briefly review the procedure to obtain the primordial scalar power spectrum for the collapse approaches described in the previous section. Afterwards, in the following sections, we will compare the predictions for the primordial power spectra resulting from the collapse models, with recent CMB data.

We begin by characterizing the CMB radiation in terms of the temperature anisotropies $\Theta(\hat{n}) \equiv \delta T / T_{0}$ of the CMB, with $T_{0}$ the mean temperature and $\delta T \equiv T \hat{n}-T_{0}$ where $T \hat{n}$ is the temperature of the CMB radiation in the direction $\hat{n}$ in the sky. The coefficients $a_{l m}$ of the spherical harmonic expansion of $\Theta(\hat{n})$ are

$$
a_{l m}=\int \Theta(\hat{n}) Y_{l m}^{\star}(\theta, \varphi) d \Omega
$$

with $\hat{n}=(\sin \theta \sin \varphi, \sin \theta \cos \varphi, \cos \theta)$ and $\theta, \varphi$ the coordinates on the celestial two-sphere. The Fourier decomposition for the temperature anisotropies can be written as follows: $\Theta(\hat{n})=\sum_{\vec{k}}\left(\Theta(\vec{k}) / L^{3}\right) e^{i \vec{k} \cdot R_{D} \hat{n}}$ with $R_{D}$ being the radius of the last scattering surface. We recall that $\Theta(\hat{n})$ is directly related to the primordial curvature perturbation. In the comoving gauge, which is the one considered in the numerical code we are going to use in the next section, the curvature perturbation is given by the field $\mathcal{R}$.

In Fourier space, the temperature anisotropies and the initial curvature perturbation are related as $\Theta(\vec{k})=T(k) \mathcal{R}_{\vec{k}}$, where $T(k)$ is the transfer function which contains the physics between the beginning of the radiation-dominated era and the present. Consequently, the coefficients $a_{l m}$, in terms of the modes $\mathcal{R}_{\vec{k}}$ can be expressed:

$$
a_{l m}=\frac{4 \pi i^{l}}{L^{3}} \sum_{\vec{k}} j_{l}\left(k R_{D}\right) Y_{l m}^{\star}(\hat{k}) T(k) \mathcal{R}_{\vec{k}},
$$

with $j_{l}\left(k R_{D}\right)$ being the spherical Bessel function of order $l$.

On the other hand, in the collapse schemes and the CSL inflationary approaches, the theoretical predictions were obtained choosing the longitudinal gauge. In that gauge, the curvature perturbation is characterized by the Newtonian potential $\Psi$. The relation between $\Psi$ and $\mathcal{R}$ is $\mathcal{R}=\Psi+(2 / 3)\left(\mathcal{H}^{-1} \Psi^{\prime}+\Psi\right) /(1+\omega)$, with $\omega \equiv P / \rho$ [56] [66]. During the inflationary epoch $\omega+1 \simeq(2 / 3) \epsilon_{1}$. In fact, for the modes of observational interest $\mathcal{R}_{\vec{k}} \simeq \Psi_{\vec{k}} / \epsilon_{1}$, with $\Psi_{\vec{k}}$ given in eq. (5). Hence, eq. (26) can be recasted as,

$$
a_{l m}=\frac{4 \pi i^{l}}{L^{3}} \sum_{\vec{k}} j_{l}\left(k R_{D}\right) Y_{l m}^{\star}(\hat{k}) T(k) \frac{\Psi_{\vec{k}}}{\epsilon_{1}} .
$$


Furthermore, using eq. (5) and the definition of the conjugated momentum of the field $\hat{y}$, the expression for the coefficients $a_{l m}$ can be expressed in the final form

$$
a_{l m}=\frac{4 \pi i^{l}}{L^{3}} \frac{H}{\sqrt{2 \epsilon_{1}} M_{P}} \sum_{\vec{k}} j_{l}\left(k R_{D}\right) Y_{l m}^{\star}(\hat{k}) T(k) \frac{\left\langle\hat{\pi}_{\vec{k}}\right\rangle}{k^{2}} .
$$

In the collapse schemes and CSL inflationary approaches, the expectation value $\left\langle\hat{\pi}_{\vec{k}}\right\rangle$ is a random variable: in the first such random variable is characterized by $x_{\vec{k}}$, while in the CSL inflationary approach, each realization of $\left\langle\hat{\pi}_{\vec{k}}\right\rangle$ corresponds to a particular post-collapse state, the stochasticity of said state is generated from the noise function $\mathcal{W}$. As a consequence, the coefficients $a_{l m}$, given in eq. [28), are, in effect, a sum of random complex numbers, just like in an effective two-dimensional random walk. Nevertheless, one cannot give a perfect estimate for the direction of the final displacement resulting from the random walk, instead, one might provide an estimate for the length of the displacement. Thus, we can obtain an estimate for the most likely value of $\left|a_{l m}\right|^{2}$, and interpret it as the theoretical prediction for the observed value. Moreover, such estimate can be obtained as follows: given that the collapse is characterized by a random process, we can consider a set of possible realizations of such process leading in each case to a specific universe. That is, we consider an imaginary ensemble of universes, each member of the ensemble is characterized by the set of random variables $\left\langle\hat{\pi}_{\vec{k}}\right\rangle$ for all $\vec{k}$. If we assume no correlation between different modes, and approximate the distribution of $\left\langle\hat{\pi}_{\vec{k}}\right\rangle$ by a Gaussian, then we can identify the most likely value $\left|a_{l m}\right|_{\text {ML }}^{2}$ with the mean value $\overline{\left|a_{l m}\right|^{2}}$ of all possible realizations, i.e., $\left|a_{l m}\right|_{\mathrm{ML}}^{2}=\overline{\left|a_{l m}\right|^{2}}$.

The quantity that is used in the statistical analysis to compare with observational data is the angular power spectrum: $C_{l}=(2 l+1)^{-1} \sum_{m}\left|a_{l m}\right|^{2}$. The previous discussion lead us to identify the observed value of $\left|a_{l m}\right|^{2}$ with the ensemble average $\overline{\left|a_{l m}\right|^{2}}$. Hence, after passing to the continuum, the theoretical angular power spectrum is

$$
C_{l}=4 \pi \int_{0}^{\infty} \frac{d k}{k} j_{l}\left(k R_{D}\right)^{2} T(k)^{2} P(k),
$$

where $P(k)$ is a function of $k$ that can be interpreted as an effective power spectrum (dimensionless), which is given by

$$
P(k)=\frac{H^{2}}{k M_{P}^{2} \epsilon_{1}} \overline{\left\langle\hat{\pi}_{\vec{k}}\right\rangle\left\langle\hat{\pi}_{\vec{k}}\right\rangle^{*}}
$$

In the latter equation it is clear that the effective power spectrum is not the same as the one in the standard approach. Indeed the latter is identified with the quantum two-point correlation function $\langle 0|\hat{\mathcal{R}}(x) \hat{\mathcal{R}}(y)| 0\rangle$, while the former, is obtained from the ensemble average of two-product expectation values $\left\langle\hat{\pi}_{\vec{k}}\right\rangle$ in the post-collapse state. The explicit form of the effective power spectrum depends on whether the collapse schemes or the CSL inflationary approach is being used 9

\section{A. Effective power spectrum in the collapse schemes approach}

In the collapse schemes approach, the evolution of the expectation value $\left\langle\hat{\pi}_{\vec{k}}(\eta)\right\rangle$, is calculated in terms of the expectations values $\left\langle\hat{\pi}_{\vec{k}}\left(\eta_{\vec{k}}^{c}\right)\right\rangle$ and $\left\langle\hat{y}_{\vec{k}}\left(\eta_{\vec{k}}^{c}\right)\right\rangle$ evaluated at the time of collapse of the mode $\vec{k}: \eta_{\vec{k}}^{c}$ (all the expectations values are taken in the post-collapse state). In particular, one obtains an expression of the form

$$
\left\langle\hat{\pi}_{\vec{k}}(\eta)\right\rangle=F\left(k \eta, z_{k}\right)\left\langle\hat{y}_{\vec{k}}\left(\eta_{\vec{k}}^{c}\right)\right\rangle+G\left(k \eta, z_{k}\right)\left\langle\hat{\pi}_{\vec{k}}\left(\eta_{\vec{k}}^{c}\right)\right\rangle
$$

where we recall that $z_{k} \equiv k \eta_{\vec{k}}^{c}$ while the explicit expressions for the functions $F$ and $G$ are given in ref. [19]. The expectation values $\left\langle\hat{\pi}_{\vec{k}}\left(\eta_{\vec{k}}^{c}\right)\right\rangle$ and $\left\langle\hat{y}_{\vec{k}}\left(\eta_{\vec{k}}^{c}\right)\right\rangle$ are characterized for each collapse scheme. In the Newtonian and Wigner schemes, the proposed characterization is shown in eqs. 111) and (14) respectively.

Given that the transfer functions $T(k)$ involved in the final expression for the angular spectrum, eq. 29, encode the post-inflationary evolution of the primordial perturbations, we evaluate $\left\langle\hat{\pi}_{\vec{k}}(\eta)\right\rangle$ at a time near the end of the inflationary regime, i.e. when $-k \eta \ll 1$. On the other hand, within our assumptions the collapse can take place at

9 A more technical presentation on how to obtain the effective power spectrum and its conceptual meaning is given in Appendix D of ref. 19. 
any time during inflation. In particular, it can occur when the proper wavelength of the mode is bigger or smaller than the Hubble radius. In this paper, we focus on the case where the proper wavelength associated to the mode is smaller than the Hubble radius, at the time of collapse; in other words $k \gg a\left(\eta_{\vec{k}}^{c}\right) H$, which is equivalent to $-k \eta_{\vec{k}}^{c} \gg 1$.

Therefore, using eqs. (30) and (31), the equivalent power spectrum that results from the collapse schemes approach is 19

$$
P(k)=A_{s} Q\left(z_{k}\right) k^{n_{s}-1}
$$

The predicted amplitude of the power spectrum is similar to the one given in the standard inflationary picture, $A_{s} \propto H^{2} / M_{P}^{2} \epsilon_{1}$. However, the collapse hypothesis modifies the prediction for the spectral index

$$
n_{s}-1=2 \epsilon_{1}-\epsilon_{2}
$$

We recall that in the standard inflationary scenario: $n_{s}-1=-2 \epsilon_{1}-\epsilon_{2}$. In addition, there is a new function of the time of collapse $Q\left(z_{k}\right)$ which is different for each collapse scheme; in the Newtonian scheme,

$$
\begin{aligned}
& Q^{\text {Newt. }}\left(z_{k}\right)=\left[1+\frac{1}{\left|z_{k}\right|^{2}}\left(-2 \nu+\frac{\Gamma(\nu+5 / 2)}{2 \Gamma(\nu+1 / 2)}\right)^{2}\right] \\
& \times\left[\cos \beta\left(\nu,\left|z_{k}\right|\right)-\frac{\sin \beta\left(\nu,\left|z_{k}\right|\right)}{2\left|z_{k}\right|} \frac{\Gamma(\nu+3 / 2)}{\Gamma(\nu-1 / 2)}\right]^{2}
\end{aligned}
$$

while in the Wigner scheme,

$$
\begin{aligned}
& Q^{\text {Wig. }}\left(z_{k}\right)=\left\{\left[\frac{2 \nu}{\left|z_{k}\right|^{3 / 2}}\left(\cos \beta\left(\nu,\left|z_{k}\right|\right)-\frac{\sin \beta\left(\nu,\left|z_{k}\right|\right)}{2\left|z_{k}\right|} \frac{\Gamma(\nu+3 / 2)}{\Gamma(\nu-1 / 2)}\right)\right.\right. \\
- & \left(\sin \beta\left(\nu,\left|z_{k}\right|\right)+\frac{\cos \beta\left(\nu,\left|z_{k}\right|\right)}{2\left|z_{k}\right|} \frac{\Gamma(\nu+5 / 2)}{\Gamma(\nu+1 / 2)}\right] \cos \Theta_{k} \\
+ & {\left.\left[\cos \beta\left(\nu,\left|z_{k}\right|\right)-\frac{\sin \beta\left(\nu,\left|z_{k}\right|\right)}{2\left|z_{k}\right|} \frac{\Gamma(\nu+3 / 2)}{\Gamma(\nu-1 / 2)}\right] \sin \Theta_{k}\right\}^{2}, }
\end{aligned}
$$

where $\nu=2-n_{s} / 2, \beta\left(\nu,\left|z_{k}\right|\right) \equiv\left|z_{k}\right|-(\pi / 2)(\nu+1 / 2)$ and $\tan 2 \Theta_{k} \simeq-4 / 3\left|z_{k}\right|$.

It follows from eq. (32) that if we consider $z_{k}$ independent of $k$, then we recover the standard shape of the spectrum, that is $P(k)^{\text {std. }} \propto k^{n_{s}-1}$. Furthermore, in previous works [18, 19], small departures from this expression of the form $z_{k}=\mathcal{A}+\mathcal{B} k$, were considered. For this choice of $z_{k}$ the collapse time of each mode reads:

$$
\eta_{c}^{k}=\frac{\mathcal{A}}{k}+\mathcal{B}
$$

where $\mathcal{A}$ is dimensionless and $\mathcal{B}$ has units of Mpc. The comparison between the primordial power spectrum, which resulted from the Newtonian/Wigner schemes, and the standard spectrum from the traditional inflationary model has been shown and discussed thoroughly in ref. 19] for different values of $\mathcal{A}$ and $\mathcal{B}$. A statistical analysis contrasting the effect of this kind of dependence of collapse time on the mode's wave number on the CMB spectrum with WMAP9 data has been performed in ref. [18]. On the other hand, in a recent work, some of us have also studied a different possibility for the dependence of collapse time on the mode's wave number which affects predominantly the low $\ell$ part of the CMB spectrum [43. Results from a Bayesian model comparison analysis indicate that the data show no preference between the Wigner collapse model and the standard $\Lambda$ CDM model. Therefore, it is interesting to analyze the effect of the dependence in eq. 36 with recent CMB data and perform a Bayesian model comparison analysis. Here, we mention that the inflationary expansion period corresponds to negative conformal time, so we choose to work with negative values for $\mathcal{A}$ and $\mathcal{B}$.

Figure 1, shows $Q(k)$, the modification of the power spectrum in the Wigner scheme for three different values of $\mathcal{B}$ and fixed $\mathcal{A}$. Recall that the $k$ dependence on $Q$ is inherited through the variable $z_{k} \equiv k \eta_{\vec{k}}^{c}$, see Eqs. 35 and 36 . Also, it should be noted that $Q(k)=$ constant means no change in the standard shape of the power spectrum. The effect of considering the Wigner collapse scheme on the primordial power spectrum induces an important modification in both the amplitude and shape of the mentioned spectrum. Besides, the intensity of the change depends on the value of $\mathcal{B}$. It follows from Figure 1 that the influence of the collapse scheme is most significant for high values of $k$, which will result in a change in the small angular scales of the CMB temperature and polarization spectrum (see Fig. 3. The same analysis can be done for the Newton collapse scheme, resulting in similar conclusions (see Ref. [19]). 


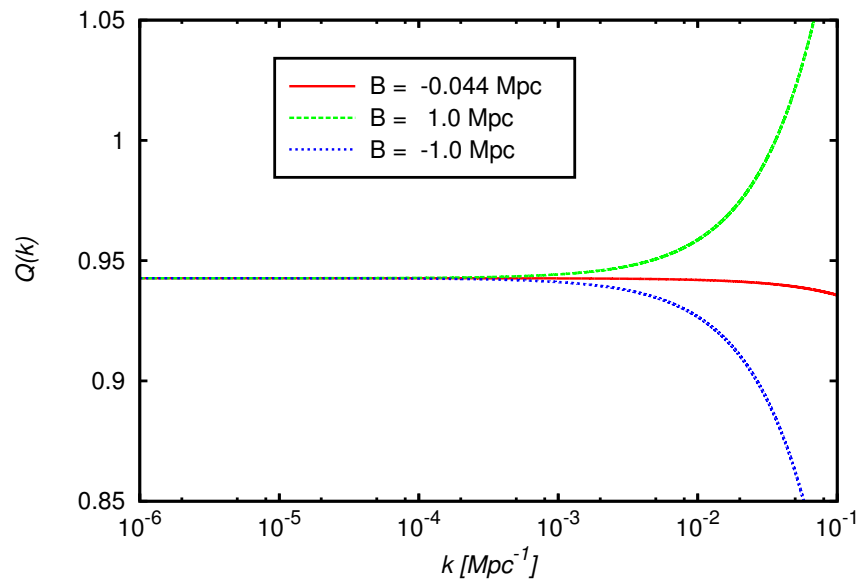

Figure 1. The function $Q(k)$ associated to the power spectrum in the Wigner collapse scheme. We have set the value $\mathcal{A}=-750$. We have considered three different values of $\mathcal{B}$ (displayed in the figure).

\section{B. Effective power spectrum in the CSL inflationary approach}

As indicated by eq. $(30)$, one needs to compute the average $\overline{\left\langle\hat{\pi}_{\vec{k}}\right\rangle\left\langle\hat{\pi}_{\vec{k}}\right\rangle^{*}}$ in order to provide a prediction for the effective power spectrum. That average is

$$
\begin{aligned}
\overline{\left\langle\hat{\pi}_{\vec{k}}\right\rangle\left\langle\hat{\pi}_{\vec{k}}\right\rangle^{*}} & =\overline{\left\langle\hat{\pi}_{\vec{k}}^{\mathrm{R}}+i \hat{\pi}_{\vec{k}}^{\mathrm{I}}\right\rangle\left\langle\hat{\pi}_{\vec{k}}^{\mathrm{R}}-i \hat{\pi}_{\vec{k}}^{\mathrm{I}}\right\rangle} \\
& =\overline{\left\langle\hat{\pi}_{\vec{k}}^{\mathrm{R}}\right\rangle^{2}}+\overline{\left\langle\hat{\pi}_{\vec{k}}^{\mathrm{I}}\right\rangle^{2}} .
\end{aligned}
$$

Furthermore, $\overline{\left\langle\hat{\pi}_{\vec{k}}^{\mathrm{R}}\right\rangle^{2}}=\overline{\left\langle\hat{\pi}_{\vec{k}}^{\mathrm{I}}\right\rangle^{2}}$. In the CSL inflationary approach, it can be shown [20] that,

$$
\overline{\left\langle\hat{\pi}_{\vec{k}}^{\mathrm{R}, \mathrm{I}}\right\rangle^{2}}=\overline{\left\langle\left(\hat{\pi}_{\vec{k}}^{\mathrm{R}, \mathrm{I}}\right)^{2}\right\rangle}-\frac{1}{\operatorname{Re}\left[A_{k}(\eta)\right]} .
$$

The quantity $(\operatorname{Re}[A(\eta)])^{-1}$ represents the standard deviation of the squared momentum. It is also the width of every packet in momentum space. The technical steps to obtain the right hand side of eq. (38) are presented in ref. [20]. However, in the present work we have generalized those steps to the quasi-de Sitter case.

In particular, we need to use the expression for $A_{k}(\eta)$, eq. (24), and find a suitable approximation for the case $-k \eta \ll 1$, i.e.

$$
\frac{1}{\operatorname{Re}\left[A_{k}(\eta)\right]} \simeq \frac{k 2^{2 \mu-2} \sin (\pi \mu) \Gamma^{2}(\mu)(-k \eta)^{-2 \mu+1}}{\pi \zeta_{k}^{2 \mu} \sin \left(2 \mu \theta_{k}+\pi \mu\right)}
$$

where we have defined $\zeta_{k} e^{i \theta_{k}} \equiv \sqrt{1-2 i \lambda_{k}}$. Additionally, the quantity $\overline{\left\langle\left(\hat{\pi}_{\vec{k}}^{\mathrm{R}, \mathrm{I}}\right)^{2}\right\rangle}$ can be approximated for the case $-k \eta \ll 1$, as

$$
\begin{aligned}
\overline{\left\langle\left(\hat{\pi}_{\vec{k}}^{\mathrm{R}, \mathrm{I}}\right)^{2}\right\rangle} & \simeq \frac{k}{\pi} 2^{2 \mu-2} \Gamma(\mu)^{2}(-k \eta)^{-2 \mu+1} \\
& \times\left[1+\lambda_{k} \sin \gamma_{k} \cos \gamma_{k}\right. \\
& \left.-\frac{\lambda_{k} k \tau}{2}\left(\frac{3}{\mu+1} \sin ^{2} \gamma_{k}+\frac{\cos ^{2} \gamma_{k}}{\mu}\right)\right]
\end{aligned}
$$

with $\gamma_{k} \equiv-k \tau-\mu \pi / 2-3 \pi / 4$. is

After inserting eqs. (39) and (40) into eq. (38), one obtains the effective power spectrum from eq. (30). The result

$$
P(k)=A_{s} C(k) k^{n_{s}-1} .
$$




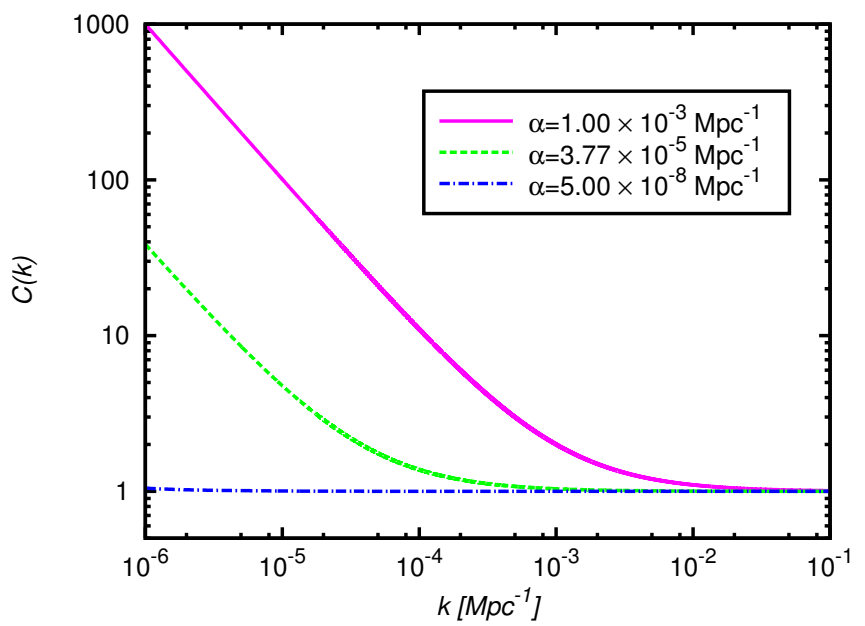

Figure 2. The function $C(k)$ associated to the power spectrum in the CSL inflationary approach. We have set the value $\lambda_{0}=1 /|\tau| \simeq 6.41 \times 10^{-9} \mathrm{Mpc}^{-1}$ and $n_{s}=0.96$. We have considered three different values of $\alpha$ (displayed in the figure). We have assumed standard values for $V_{0}$ and $N$.

As in the collapse schemes approach, the amplitude predicted in the CSL approach is the same as in the standard picture $A_{s} \propto H^{2} / M_{P}^{2} \epsilon_{1}$. Also, the prediction for the scalar spectral index is different from the standard case, but identical to that obtained for the collapse schemes approach: $n_{s}-1=2 \epsilon_{1}-\epsilon_{2}$. On the other hand, the function $C(k)$ reads

$$
\begin{aligned}
C(k) & \equiv 1+\lambda_{k} k|\tau|+\lambda_{k} \cos (k|\tau|) \sin (k|\tau|) \\
& -\frac{1}{\zeta_{k}^{2 n_{s}-1} \cos \left[\left(2-n_{s}\right) \theta_{k}\right]},
\end{aligned}
$$

where

$$
\zeta_{k} \equiv\left(1+4 \lambda_{k}^{2}\right)^{1 / 4}, \quad \theta_{k} \equiv-\frac{1}{2} \arctan \left(2 \lambda_{k}\right)
$$

It follows from the latter that when $\lambda_{k}=\frac{\lambda_{0}}{k}$ the primordial power spectrum becomes nearly scale invariant, since the most important dependence on $k$ arises from the second term. We have checked that the changes resulting from the oscillatory terms of eq. 42 do not produce important effects in the spectrum. Furthermore, it has been shown that, for the exact the Sitter case the CMB spectrum is not sensitive to the value of $\lambda_{0}$ [67. We have also verified that this is the case for the nearly invariant de Sitter case analyzed in this paper. Furthermore, just as in the collapse schemes approach, one does not expect an exact $1 / k$ dependence of $\lambda_{k}$. Thus we proceed to explore possible effects on the shape of the primordial power spectrum that would result from the following modified dependence:

$$
\lambda_{k} \equiv \lambda_{0}\left(\frac{1}{k}+\frac{\alpha}{k^{2}}\right)
$$

where we have introduced an extra parameter $\alpha$. The $\alpha / k^{2}$ term is motivated by the findings of a previous work [43]. In such a work, a similar modified dependence was considered in the context of the collapse schemes approach. The study indicated a similar Bayesian evidence as the standard $\Lambda$ CDM model.

Figure 2 shows the resulting function $C(k)$ for three different values of $\alpha$. Note that $C(k)=1$ means no modification on the standard shape of the power spectrum. We consider $\lambda_{0}=\frac{1}{\tau}$, where $\tau$ depends on two main quantities: the characteristic energy scale of inflation $V_{0}$ and the total number of e-folds of inflation $N$. We note that the effect of considering the CSL model results in an important departure in both the amplitude and shape of the large scale of the primordial power spectrum, with the intensity depending on the value of $\alpha$. The mentioned difference in shape between the standard primordial power spectrum and the one resulting from the CSL collapse model is most relevant for the lower values of $k$. On the other hand, $\lambda_{k}$ must be positive and this requirement implies $\alpha>-10^{-6}$ for the relevant $k$ values. 


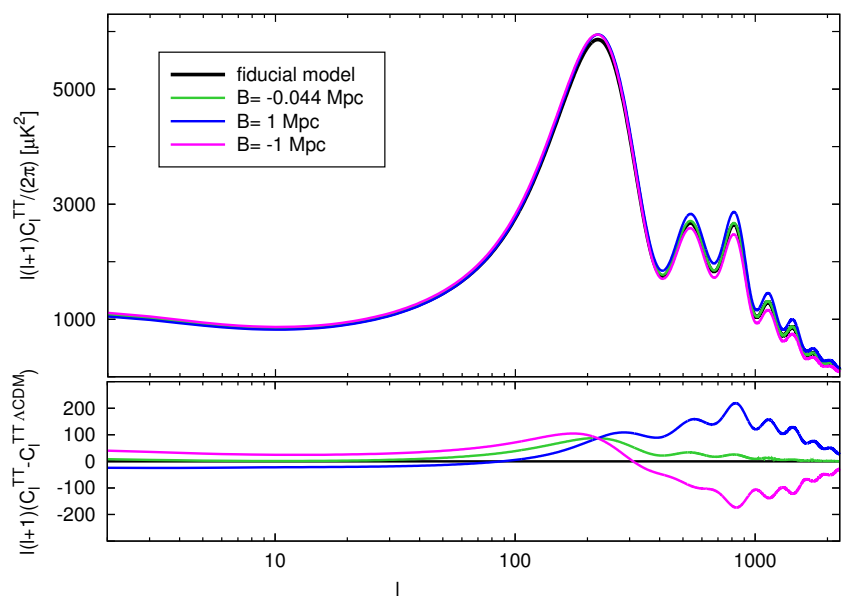

Figure 3. The temperature auto-correlation function and differential plot respect to the fiducial model for the Wigner scheme model using $\mathcal{A}=-750$ and different values of $\mathcal{B}$. All models are normalized to the maximum of the first peak of the fiducial model.

\section{Effects of the self-induced collapse on the CMB spectrum}

Next, we explore the effects on the CMB spectrum, when incorporating the self-induced collapse hypothesis within the various approaches considered in this paper. Hereafter, we assume as a reference the $\Lambda$ CDM best-fit model reported by the Planck Collaboration (2015) 68, ${ }^{10}$ As regards the collapse schemes, it has already been shown in previous works [18, 19, 43] that if $\mathcal{B}=0$ then the standard primordial power spectrum is recovered except for an overall normalization factor, just as it does any change in the collapse parameter $\mathcal{A}$.

Figures 3 and 4 show the CMB temperature and polarization spectra for the Wigner scheme model using a fixed $\mathcal{A}$ value and different values of $\mathcal{B}$. We choose to fix $\mathcal{A}$ to an "appropriate" value (we stress that a change in $\mathcal{A}$ affects the power spectrum just by an overall normalization) so the value of $A_{s}$ that gives a good fit to the CMB data is the closest to the standard $\Lambda \mathrm{CDM}$ value. At the same time, we chose the values of the cosmological parameters for the collapse scheme models to be the same as the ones of the fiducial model. We noted an increase in the value of the secondary peaks of the temperature power spectrum and a decrease in the values at the valleys for all cases, with the magnitude of the changes depending on the value of $\mathcal{B}$. There is also an increase in the height of the peaks in the EE spectrum, with increasing values of $\mathcal{B}$ while for the TE cross correlation temperature we only noted a change in the height of the valleys with the the intensity depending on the value of $\mathcal{B}$. A similar analysis was made for the Newtonian scheme model; the effects on the CMB spectrum were similar, with the main difference being that in this scheme the results are less sensitive to changes in $\mathcal{B}[19]$.

For the CSL collapse model, we note that a change in the parameter $\alpha$ mainly affects the low multipole region as can be appreciated in figure 5. However, we also observe a very small change in the height of the peaks (with respect to the fiducial model) for the E-mode spectrum while the temperature-polarization spectrum shows no changes with respect to the fiducial model (see figure 6). These effects are similar to the ones found in collapse schemes models with $\eta_{c}^{k}=\frac{\mathcal{A}}{k}+\frac{\mathcal{B}}{k^{2}}$ (see ref. [43]).

\section{ANALYSIS METHOD}

In this work we consider the two collapse schemes models, i.e. the Newtonian and the Wigner schemes, the CSL collapse model and the $\Lambda \mathrm{CDM}$ one (that we use as a reference).

In our analysis, we vary the usual cosmological parameters, namely, the physical baryon density, $\Omega_{b} h^{2}$, the physical cold dark matter density, $\Omega_{c} h^{2}$, the ratio between the sound horizon and the angular diameter distance at decoupling, $\theta$, the optical depth, $\tau$, the primordial amplitude, $A_{s}$, the spectral index $n_{s}$ and the additional collapse parameter $\mathcal{A}$,

10 We use the values obtained using the $\mathrm{TT}+$ lowP data. 

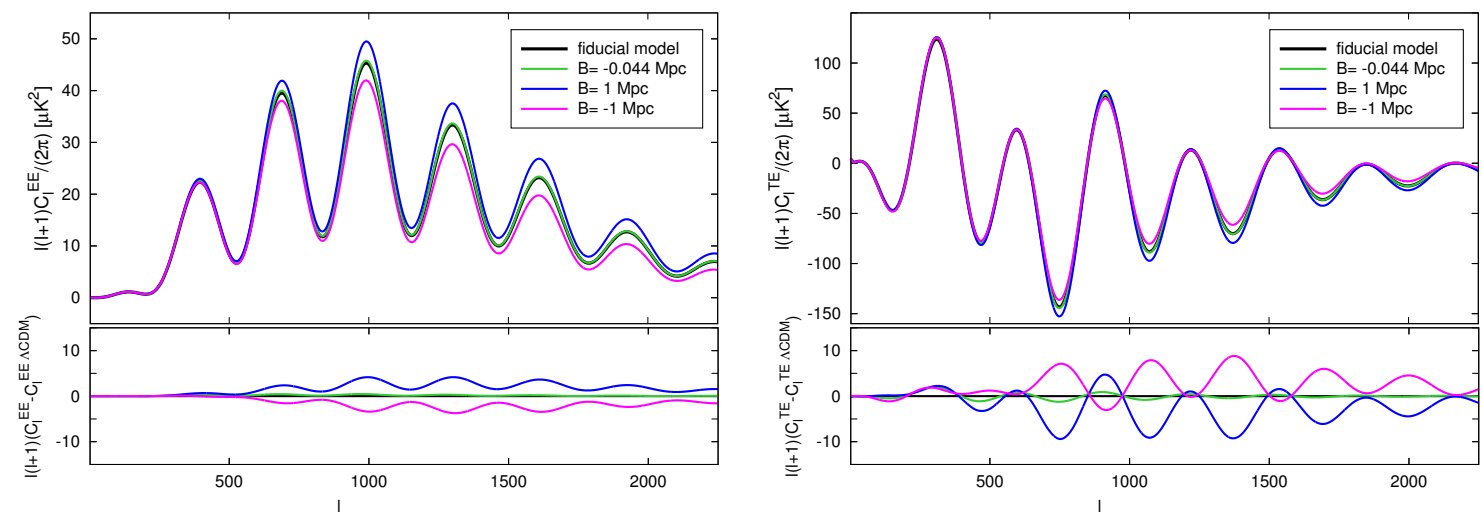

Figure 4. Predictions for the Wigner scheme model using $\mathcal{A}=-750$ and different values of $\mathcal{B}$. Left: The E-mode (EE) auto-correlation function and differential plot respect to the fiducial model $\mathrm{f}$ Right: The temperature-E mode polarization (TE) cross correlation power spectrum and differential plot respect to the fiducial model. All models are normalized to the maximum of the first peak of the fiducial model.

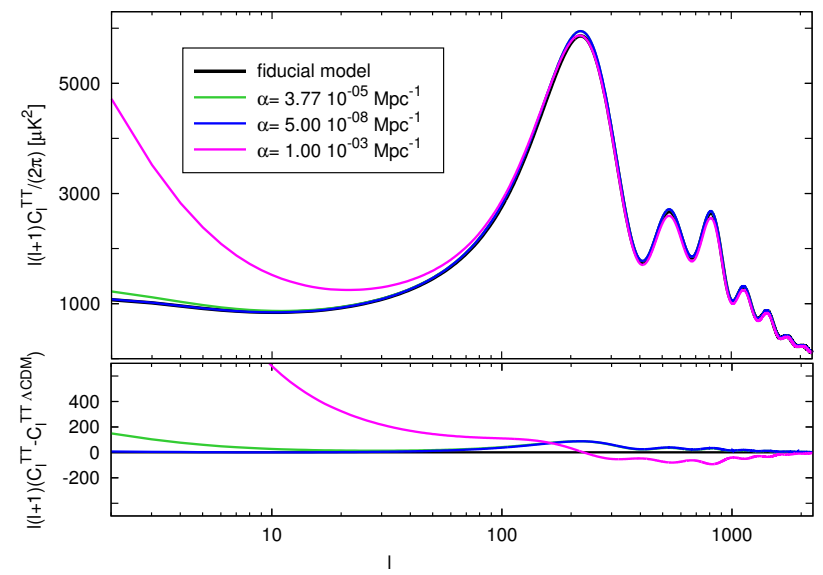

Figure 5. The temperature (TT) auto-correlation power spectrum and differential plot respect to the fiducial model for the CSL model for different values of $\alpha$. All models are normalized to the maximum of the first peak of the fiducial model.
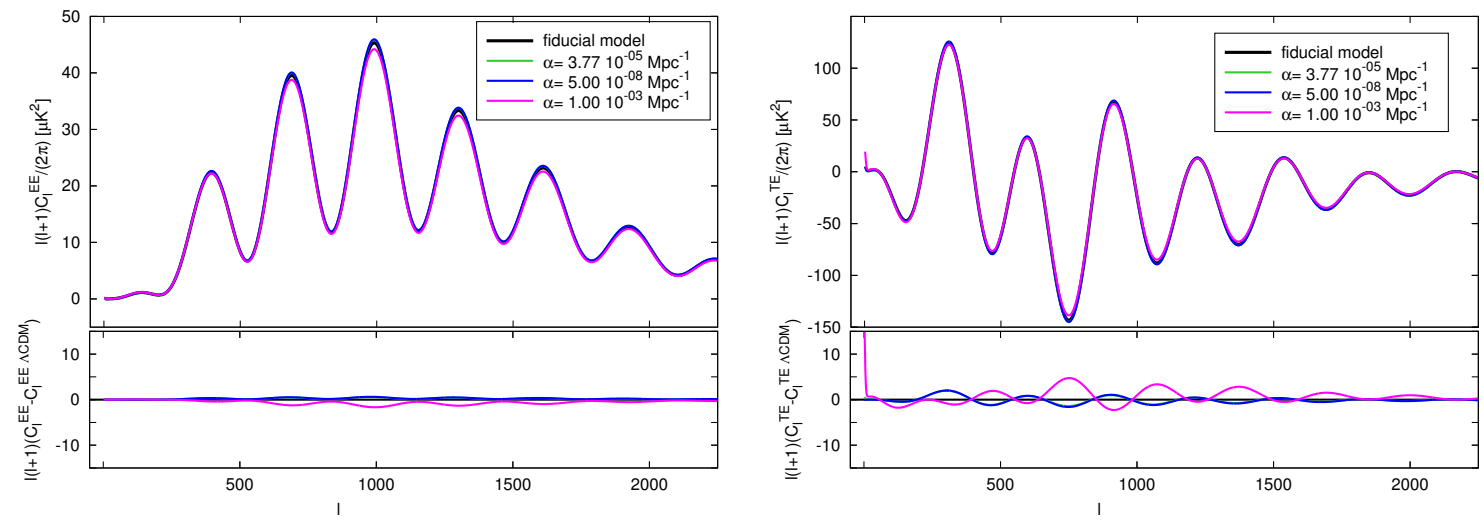

Figure 6. Predictions for the CSL collapse model for different values of $\alpha$. Left: The E-mode polarization (EE) auto-correlation power spectrum and differential plot respect to the fiducial model Right: The temperature-E mode polarization (TE) cross correlation power spectrum and differential plot respect to the fiducial model. All models are normalized to the maximum of the first peak of the fiducial model. 
$\mathcal{B}$ and $\alpha$. We also vary the nuisance foreground parameters [69] and consider purely adiabatic initial conditions. The sum of neutrino masses is fixed to $0.06 \mathrm{eV}$, and we limit the analysis to scalar perturbations with $k_{*}=0.05 \mathrm{Mpc}^{-1}$.

We work with flat priors for the cosmological and collapse parameters, and choose to fix the collapse parameter $\mathcal{A}$. As has been discussed in the previous section, the $\mathcal{A}$ parameter just affects the primordial spectrum as a change in the amplitude; therefore it is highly degenerate with the $A_{s}$ parameter. Thus, we have tested several values for the $\mathcal{A}$ parameter, and fixed a value which satisfies the condition for the conformal collapse time $\eta_{c}^{k}<0$ and minimizes the variation of the $A_{s}$ from the $\Lambda \mathrm{CDM}$ model value. (We chose $\mathcal{A}=-750$ for the Wigner scheme and $\mathcal{A}=-600$ for the Newton scheme.)

In order to compare the quantum collapse inflationary models with recent CMB data, we need to compute CMB anisotropies including the modifications in the primordial power spectrum. For this, we modify the public available Code for Anisotropies in the Microwave Background (CAMB) [70. In our analysis, we perform a Monte Carlo Markov chain exploration of the parameters space using the available package CosmoMC [71] and implement the nested sampling algorithm of Multinest code [72-74 to obtain the Bayesian evidence of the model. For the Bayesian analysis we use the most accurate Importance Nested Sampling (INS) [74, 75] instead of the vanilla Nested Sampling (NS), and we require a INS Global Log-Evidence error $\leq 0.02$.

For the data analysis, we use the current Planck Collaboration release (2015) 68 and BAO data. In particular, we consider the high- $\ell$ Planck temperature data from the 100-,143-, and 217-GHz half-mission T maps, and the low- $\ell$ data by the joint TT,EE,BB and TE likelihood. Also, we consider Baryonic Acoustic Oscillation data by the 6dF Galaxy Survey (6dFGS) [76], SDSS DR7 Main Galaxy Sample (SDSS-MGS) galaxies [77, BOSS galaxy samples, LOWZ and CMASS [78].

We perform an appropriate comparison between the quantum collapse inflationary models and the standard $\Lambda$ CDM model predictions using the Bayesian model comparison. This is a powerful tool to favor the models that fit well the data exhibiting strong predictivity, while models with a large number of free parameters, not required by the data, are penalised for the wasted parameter space (we refer the reader to some recent employ on cosmological models [79 84]).

We can write the Bayes factor $B_{i j}=\frac{\mathcal{E}_{M_{i}}}{\mathcal{E}_{M_{j}}}$, where $\mathcal{E}_{M_{i}}$ is the evidence of the analysed model and $\mathcal{E}_{M_{j}}$ the reference model one. The usual scale employed to judge the Bayes factor is the Jeffreys scale [85, 86], that is

\begin{tabular}{cccc}
$\ln \mathcal{B}_{i j}$ & Odds & Probability & Notes \\
\hline$<1$ & $<3: 1$ & $<0.750$ & inconclusive \\
1 & $\sim 3: 1$ & 0.750 & weak evidence \\
2.5 & $\sim 12: 1$ & 0.923 & moderate evidence \\
5 & $\sim 150: 1$ & 0.993 & strong evidence
\end{tabular}

Note that negative Bayes factor value means support in favor of the reference model $j$.

\section{RESULTS}

Before presenting our results, let us draw attention on the different degeneracy between $n_{s}$ and $\mathcal{B}$ for the two collapse schemes models analysed. In figure 7, we can observe that, for the Newtonian scheme (green curve), increasing values of $n_{s}$ allow for higher values of $\mathcal{B}$; while in the Wigner scheme (blue curve) for crescent values of $n_{s}$, lower values of $\mathcal{B}$ are preferred by the data. Furthermore, the Newtonian scheme allows for high values of the parameter $n_{s}$ (until the unity) and it is interesting in the context of the so-called $H_{0}$ tension. Indeed, the degeneracy between the spectral index parameter and the local value of the Hubble constant, (i.e. higher value of $n_{s}$ produces an increase in the value of $H_{0}$ ), reduces the tension between the $H_{0}$ value derived by CMB analysis and the local measurements of Riess et al. from the Hubble Space Telescole (HST) 87] (see refs. 88 91] for recent discussions about the $H_{0}$ tension).

We present the cosmological analysis for the collapse schemes models in tables I and II We note that the resulting constraints on the parameters' values are in general agreement with those obtained for the $\Lambda$ CDM model. However, it should be noted that $n_{s}$ and $A_{s}$ are less constrained than in the standard model due to the degeneration of these parameters with $\mathcal{B}$. On the other hand, while the main value of the primordial amplitude is in agreement with that obtained for the $\Lambda$ CDM model in the Wigner model, we note a shift in the one obtained for the Newtonian scheme. Furthermore, we also note that the constraints on $\mathcal{B}$ are narrower in the Wigner scheme than the Newtonian one. This reflects the increased sensibility of the observational predictions of this model to small variations of the $\mathcal{B}$ parameter value.

In the last lines of tables $\mathrm{I}$ and $\mathrm{II}$ we report the $\Delta \chi^{2}$ and the Bayes factor $\ln \mathcal{B}_{i j}$ for the models with respect to the standard cosmological one. For the Newtonian scheme, the $\chi^{2}$ value is better than the $\Lambda$ CDM one of one point while in the Wigner scheme the improvement over the $\Lambda \mathrm{CDM}$ model is 1.9. However, the data show a moderate preference for the $\Lambda \mathrm{CDM}$ model over the Newtonian model and a weak preference over the Wigner one. This is due to the spread in the non-gaussian profile of the posterior probability distributions for $\mathcal{B}$. 
In order to improve these results, we also compared the predictions of the collapse scheme model with the BAO data. Indeed, the imprint of baryon acoustic oscillations in large-scale structure are a powerful tool for mapping out the cosmic expansion history and constrain the cosmological parameters. Given that the Wigner scheme is more sensitive to changes with the $\mathcal{B}$ parameter, and also shows a better Bayesian evidence than the Newtonian scheme, we select it for this second analysis. In table III we report the results for the joint CMB and BAO data set. We can see that the values of the cosmological parameters are more strongly constrained but, at the same time the constraints on $\mathcal{B}$ show almost no difference when the BAO data are considered (see also the figure 8). Furthermore, the data show moderate Bayesian preference for the Wigner scheme model over the $\Lambda \mathrm{CDM}$ model for the $\mathrm{CMB}+\mathrm{BAO}$ data. We stress that, comparing with the Bayesian evidence of the tables I and II] the Wigner scheme shows very closed value while the $\Lambda$ CDM model gets a value worst of about 6 points. This means that the improving in the $B_{i j}$ is mainly due to a worst fit of the $\Lambda \mathrm{CDM}$ data of the new dataset, while the Wigner scheme proves to be more conservative.

Now, we look to the cosmological analysis of the CSL collapse model. The results are in table [V] where we note an excellent agreement between the parameters values obtained from the analysis of the CSL collapse and the $\Lambda$ CDM model. Moreover, in this case there is no degeneration between $n_{s}$ and the $\alpha$ collapse parameter (see figure 9 p and no increase of $n_{s}$ constraints is encountered for this model. On the other hand, the $\alpha$ parameter is not well constrained, and just an upper bound was obtained from the statistical analysis. In this case, we do not analyze the CSL model using $\mathrm{BAO}$ data since the theoretical prediction of this model differs from the standard model one only at very large angular scales. On the other hand, it is well known that BAO bumps are observed only at low angular scales, which means that the BAO data provide no useful information from large angular scales [92. Finally, we note that the data show the same Bayesian preference for this model and the standard cosmological one.

Table I. $68 \%$ confidence limits for the cosmological and collapse scheme parameters. The first columns-block refer to the minimal $\Lambda \mathrm{CDM}$ model; the second block shows the constraints on the Newtonian models; $\Delta \chi_{\text {best }}^{2}=\chi_{\text {best }}^{2}(\Lambda C D M)-$ $\chi_{\text {best }}^{2}\left(\right.$ collapse model); For $\ln B_{i j}$, the reference model is $\Lambda$ CDM.

\begin{tabular}{|c|c|c|c|c|}
\hline Parameter & $\begin{array}{l}\Lambda \text { CDI } \\
\text { mean }\end{array}$ & bestfit & $\begin{array}{c}\text { Newton } \\
\text { mean }\end{array}$ & bestfit \\
\hline $100 \Omega_{b} h^{2}$ & $2.223 \pm 0.023$ & 2.218 & $2.231 \pm 0.035$ & 2.239 \\
\hline$\Omega_{c} h^{2}$ & $0.1197 \pm 0.0022$ & 0.1199 & $0.1194 \pm 0.0023$ & 0.1178 \\
\hline $100 \theta$ & $1.04087 \pm 0.00048$ & 1.04070 & $1.04094 \pm 0.00050$ & 1.04118 \\
\hline$\tau$ & $0.078 \pm 0.020$ & 0.082 & $0.078 \pm 0.021$ & 0.089 \\
\hline$n_{s}$ & $0.9656 \pm 0.0064$ & 0.9640 & $0.9670 \pm 0.0147$ & 0.9802 \\
\hline $\ln 10^{10} A_{s}{ }^{a}$ & $3.091 \pm 0.037$ & 3.100 & $4.108 \pm 0.042$ & 4.128 \\
\hline $\mathcal{B}$ & - & - & $0.050_{-0.311}^{+0.350}$ & 0.206 \\
\hline$\overline{\Delta \chi_{\text {best }}^{2}}$ & & - & & 1 \\
\hline $\ln B_{i j}$ & & - & & -3.33 \\
\hline
\end{tabular}

${ }^{\mathrm{a}} k_{0}=0.05 \mathrm{Mpc}^{-1}$.

Table II. $68 \%$ confidence limits for the cosmological and collapse scheme parameters. The first columns-block refer to the minimal $\Lambda$ CDM model; the second block shows the constraint on the Wigner scheme models; $\Delta \chi_{\text {best }}^{2}=\chi_{\text {best }}^{2}(\Lambda C D M)-$ $\chi_{\text {best }}^{2}$ (collapse model); For $\ln B_{i j}$, the reference model is $\Lambda$ CDM.

\begin{tabular}{|c|c|c|c|c|}
\hline Parameter & $\begin{array}{c}\Lambda \mathbf{C D} \\
\text { mean }\end{array}$ & bestfit & $\begin{array}{c}\text { Wigne } \\
\text { mean }\end{array}$ & bestfit \\
\hline$\overline{100 \Omega_{b} h^{2}}$ & $2.223 \pm 0.023$ & 2.218 & $2.231 \pm 0.031$ & 2.253 \\
\hline$\Omega_{c} h^{2}$ & $0.1197 \pm 0.0022$ & 0.1199 & $0.1194 \pm 0.0023$ & 0.1192 \\
\hline $100 \theta$ & $1.04087 \pm 0.00048$ & 1.04070 & $1.04093 \pm 0.00049$ & 1.04105 \\
\hline$\tau$ & $0.078 \pm 0.020$ & 0.082 & $0.080 \pm 0.021$ & 0.077 \\
\hline$n_{s}$ & $0.9656 \pm 0.0064$ & 0.9640 & $0.9700 \pm 0.0157$ & 0.9778 \\
\hline $\ln 10^{10} A_{s}{ }^{\mathrm{a}}$ & $3.091 \pm 0.037$ & 3.100 & $3.065 \pm 0.056$ & 3.080 \\
\hline $\mathcal{B}$ & - & - & $-0.037_{-0.160}^{+0.084}$ & -0.139 \\
\hline$\overline{\overline{\Delta \chi_{\text {best }}^{2}}}$ & & - & & 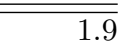 \\
\hline $\ln B_{i j}$ & & - & & -1.44 \\
\hline
\end{tabular}


Table III. $68 \%$ confidence limits for the cosmological and collapse scheme parameters using CMB and BAO data. The first columns-block refer to the minimal $\Lambda \mathrm{CDM}$ model; the second block shows the constraint on the Wigner scheme models; $\Delta \chi_{\text {best }}^{2}=\chi_{\text {best }}^{2}(\Lambda C D M)-\chi_{\text {best }}^{2}($ collapse model $)$; For $\ln B_{i j}$, the reference model is $\Lambda$ CDM.

\begin{tabular}{|c|c|c|c|c|}
\hline \multirow[b]{2}{*}{ Parameter } & \multicolumn{2}{|c|}{$\Lambda$ CDM model } & \multicolumn{2}{|c|}{ Wigner-scheme } \\
\hline & mean & bestfit & mean & bestfit \\
\hline $100 \Omega_{b} h^{2}$ & $2.233 \pm 0.020$ & 2.234 & $2.243 \pm 0.025$ & 2.256 \\
\hline$\Omega_{c} h^{2}$ & $0.1181 \pm 0.0012$ & 0.1175 & $0.1180 \pm 0.0012$ & 0.1180 \\
\hline $100 \theta$ & $1.04111 \pm 0.00041$ & 1.04089 & $1.04112 \pm 0.00042$ & 1.04106 \\
\hline$\tau$ & $0.084 \pm 0.018$ & 0.091 & $0.086 \pm 0.019$ & 0.102 \\
\hline$n_{s}$ & $0.9696 \pm 0.0043$ & 0.9692 & $0.9752_{-0.0090}^{+0.0132}$ & 0.9819 \\
\hline $\ln 10^{10} A_{s}{ }^{a}$ & $3.099 \pm 0.035$ & 3.110 & $3.082 \pm 0.050$ & 3.127 \\
\hline $\mathcal{B}$ & - & - & $-0.058_{-0.144}^{+0.071}$ & -0.134 \\
\hline$\overline{\Delta \chi_{\text {best }}^{2}}$ & - & & & 0.3 \\
\hline $\ln B_{i j}$ & & - & & 2.96 \\
\hline
\end{tabular}

${ }^{\mathrm{a}} k_{0}=0.05 \mathrm{Mpc}^{-1}$.

Table IV. $68 \%$ confidence limits for the cosmological and CSL collapse model using CMB data. The first columns-block refer to the minimal $\Lambda$ CDM model; the second block shows the constraint on the CSL collapse model; $\Delta \chi_{b e s t}^{2}=\chi_{\text {best }}^{2}(\Lambda C D M)-$ $\chi_{\text {best }}^{2}$ (collapse model); For $\ln B_{i j}$, the reference model is $\Lambda$ CDM.

\begin{tabular}{|c|c|c|c|c|}
\hline \multirow[b]{2}{*}{ Parameter } & \multicolumn{2}{|c|}{$\Lambda$ CDM model } & \multicolumn{2}{|c|}{$C S L$ model } \\
\hline & mean & bestfit & mean & bestfit \\
\hline $100 \Omega_{b} h^{2}$ & $2.223 \pm 0.023$ & 2.218 & $2.222 \pm 0.023$ & 2.234 \\
\hline$\Omega_{c} h^{2}$ & $0.1197 \pm 0.0022$ & 0.1199 & $0.1197 \pm 0.0022$ & 0.1192 \\
\hline $100 \theta$ & $1.04087 \pm 0.00048$ & 1.04070 & $1.04087 \pm 0.00048$ & 1.04064 \\
\hline$\tau$ & $0.078 \pm 0.020$ & 0.082 & $0.075 \pm 0.019$ & 0.087 \\
\hline$n_{s}$ & $0.9656 \pm 0.0064$ & 0.9640 & $0.9667 \pm 0.0062$ & 0.9674 \\
\hline $\ln 10^{10} A_{s} a^{a}$ & $3.091 \pm 0.037$ & 3.100 & $3.082 \pm 0.037$ & 3.112 \\
\hline $10^{5} \alpha$ & - & - & $<4.3$ & 0.95 \\
\hline $\begin{array}{l}\Delta \chi_{\text {best }}^{2} \\
\ln B_{i j}\end{array}$ & & & & $\begin{array}{r}-0.622 \\
-0.3 \\
\end{array}$ \\
\hline
\end{tabular}

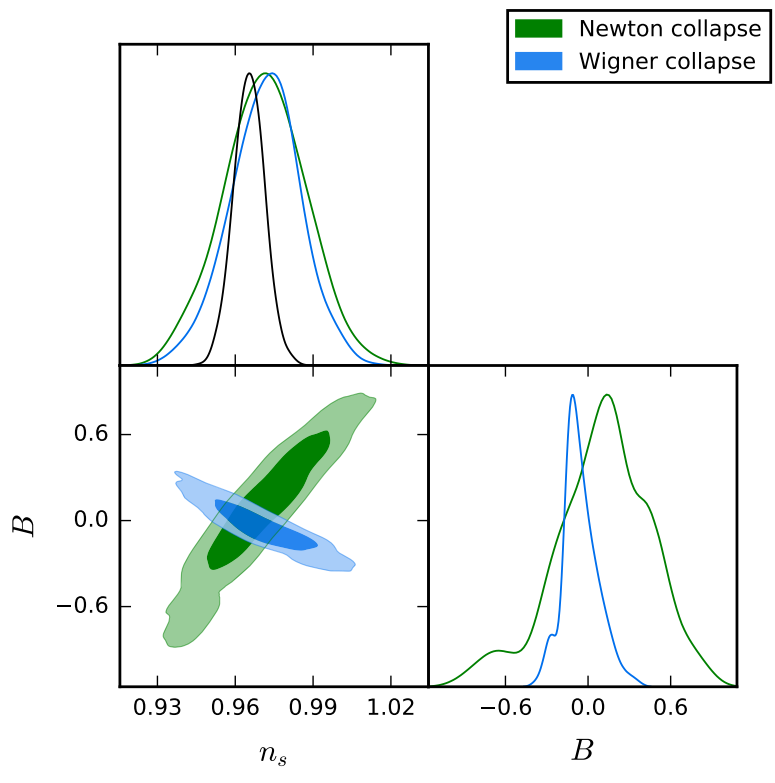

Figure 7. $68 \%$ and $95 \%$ confidence regions in the $n_{s}-B$ plane for the Newtonian (green curve) and Wigner (blue curve) collapse model. Results using the CMB data. The black curve refers to the $\Lambda$ CDM model. 


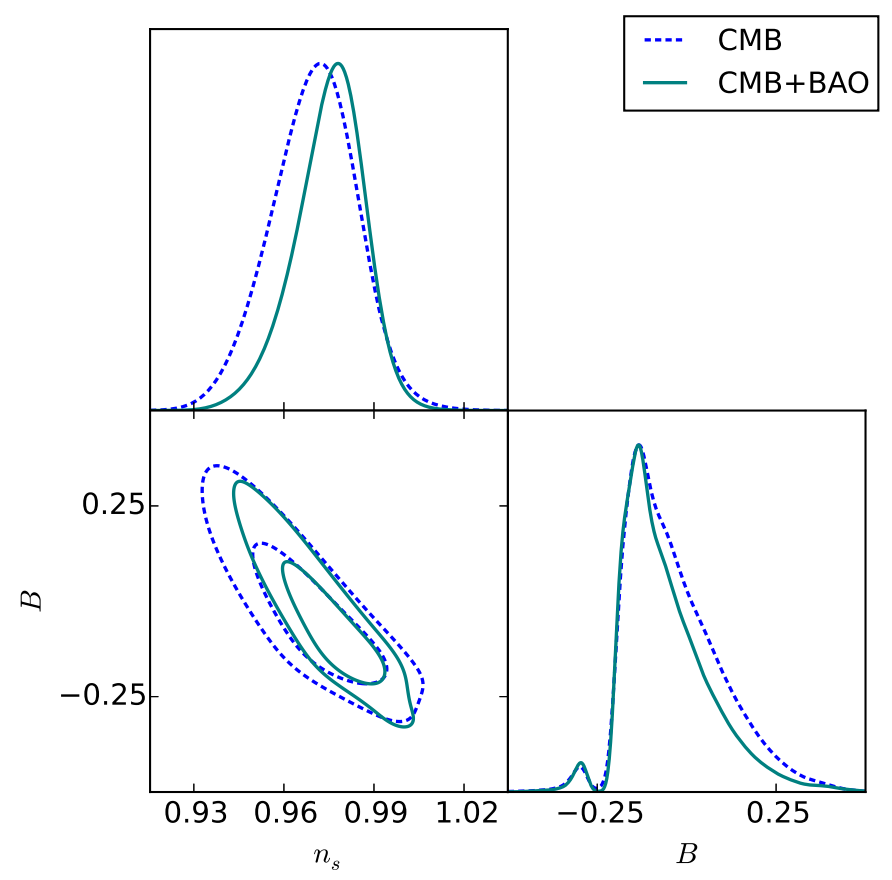

Figure 8. $68 \%$ and $95 \%$ confidence regions in the $n_{s}-B$ plane for the Wigner collapse model . One dimensional probability densities for the $B$ and $n_{s}$ parameter of the CSL collapse model. Results using only CMB and CMB $+\mathrm{BAO}$ data are shown.

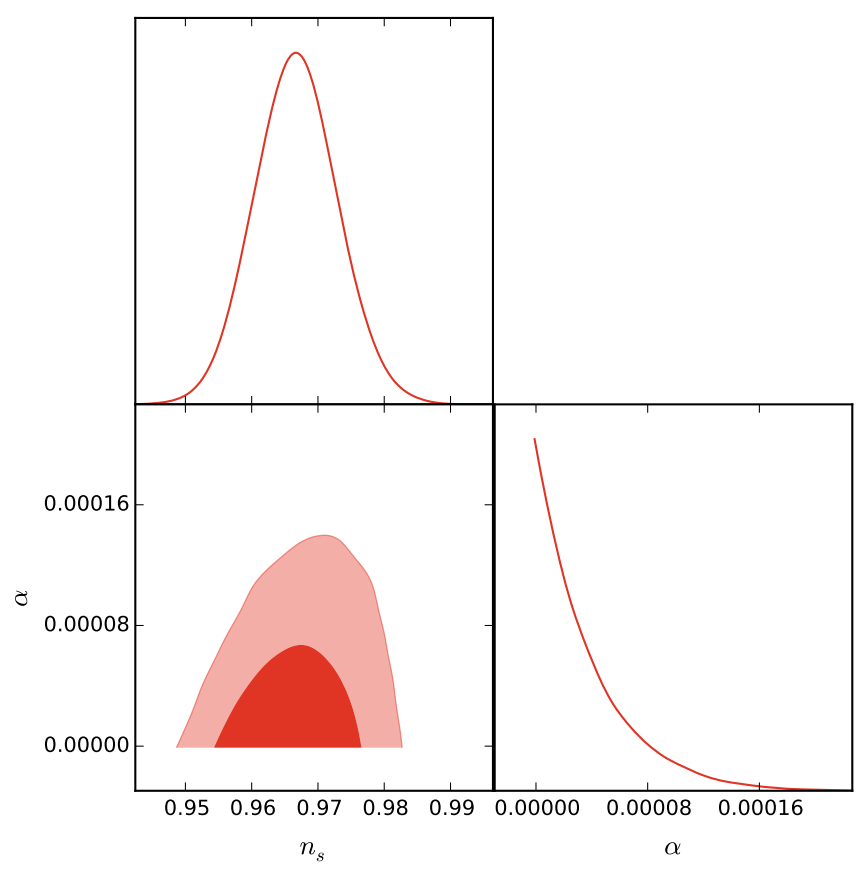

Figure 9. $68 \%$ and $95 \%$ confidence regions in the $n_{s}-B$ plane for the CSL collapse model . One dimensional probability densities for the $B$ and $n_{s}$ parameter of the CSL collapse model. 


\section{CONCLUSIONS}

In this paper, we have studied the phenomenological predictions of two different collapse proposals: the collapse scheme approach and the Continuous Spontaneous Localization inflationary collapse approach. For the former, we have considered the Newtonian and Wigner collapse schemes with the collapse time $\eta_{\vec{k}}^{c}=\mathcal{A} / k+\mathcal{B}$. For the latter, we have considered the conjugated momentum of the inflaton field as the collapse operator.

We have performed a statistical analysis in order to compare the predictions of the theoretical models with recent $\mathrm{CMB}$ and BAO data. Our findings indicate that collapse inflationary models are compatible, for the appropriate choice of the values of the free parameters with recent $\mathrm{CMB}$ and BAO data. Furthermore, for the collapse schemes considered in this work we have obtained stringent bounds on the collapse parameter $\mathcal{B}$ which characterizes the dynamics of collapse time of each mode $\eta_{\vec{k}}^{c}$. We have also obtained an upper bound on the parameter $\alpha$ of the CSL model which is related to the strength of the collapse. In addition, the values obtained for the cosmological parameters are consistent with those obtained by the Planck collaboration assuming a standard inflationary scenario. On the other hand, the constraints obtained for $n_{s}$, within the collapse schemes approach, are less stringent than those obtained in the context of the standard inflationary scenario. As a consequence, inflationary potentials that were discarded in such a context, could be reconsidered in the collapse proposal. Finally, results from the Bayesian model comparison method show a preference of the Wigner collapse model over the reference model when BAO data are included in the analysis, while there is no such preference when just the CMB data are considered. Moreover, the CSL collapse model gives the same Bayesian evidence as the standard $\Lambda$ CDM model, while the latter is preferred over the Newtonian scheme collapse model.

\section{ACKNOWLEDGEMENTS}

The authors acknowledge the use of the supercluster Mitzli at UNAM for the statistical analyses and thank the people of DGSCA-UNAM for computational and technical support. MB acknowledges financial support from the Fundação Carlos Chagas Filho de Amparo à Pesquisa do Estado do Rio de Janeiro (FAPERJ - fellowship Nota 10), and is also supported by INFN, Naples section, QGSKY project. MPP, GL and SL are supported by PIP 11220120100504 CONICET and by the National Agency for the Promotion of Science and Technology (ANPCYT) of Argentina grant PICT-2016-0081; and by grant G140 from UNLP. DS acknowledges partial financial support from DGAPA-UNAM project IG100316 and by CONACYT project 101712, the FAE-Network of CONACYT, as well as the sabbatical fellowships from CO-MEX-US (Fullbright-Garcia Robles) and from DGAPA-UNAM (Paspa). The authors acknowledge the use of CosmoMC [71] and Multinest code [72 74].

[1] A. A. Starobinsky, Phys. Lett. B91, 99 (1980)

[2] A. H. Guth, Phys. Rev. D23, 347 (1981)

[3] A. D. Linde, Second Seminar on Quantum Gravity Moscow, USSR, October 13-15, 1981, Phys. Lett. 108B, 389 (1982)

[4] A. Albrecht and P. J. Steinhardt, Phys. Rev. Lett. 48, 1220 (1982)

[5] V. F. Mukhanov and G. V. Chibisov, JETP Lett. 33, 532 (1981), [Pisma Zh. Eksp. Teor. Fiz.33,549(1981)].

[6] V. F. Mukhanov and G. V. Chibisov, Sov. Phys. JETP 56, 258 (1982), [Zh. Eksp. Teor. Fiz.83,475(1982)].

[7] A. A. Starobinsky, Phys. Lett. 117B, 175 (1982)

[8] A. H. Guth and S. Y. Pi, Phys. Rev. Lett. 49, 1110 (1982)

[9] S. W. Hawking, Phys. Lett. 115B, 295 (1982).

[10] J. M. Bardeen, P. J. Steinhardt, and M. S. Turner, Phys. Rev. D28, 679 (1983)

[11] A. Perez, H. Sahlmann, and D. Sudarsky, Classical and Quantum Gravity 23, 2317 (2005), gr-qc/0508100

[12] D. Sudarsky, International Journal of Modern Physics D 20, 509 (2011), arXiv:0906.0315 [gr-qc].

[13] S. Landau, G. León, and D. Sudarsky, Physical Review D 88, 023526 (2013), arXiv:1107.3054 [astro-ph.CO]

[14] A. de Unánue and D. Sudarsky, Physical Review D 78, 043510 (2008), arXiv:arXiv:0801.4702

[15] G. León and D. Sudarsky, Classical and Quantum Gravity 27, 225017 (2010) arXiv:1003.5950 [gr-qc]

[16] A. Diez-Tejedor and D. Sudarsky, JCAP 7, 045 (2012), arXiv:1108.4928 [gr-qc]

[17] G. León, A. De Unánue, and D. Sudarsky, Classical and Quantum Gravity 28, 155010 (2011), arXiv:1012.2419 [gr-qc].

[18] S. J. Landau, C. G. Scóccola, and D. Sudarsky, Physical Review D 85, 123001 (2012) arXiv:1112.1830 [astro-ph.CO].

[19] G. León, S. J. Landau, and M. P. Piccirilli, European Physical Journal C 75, 393 (2015), arXiv:1502.00921 [gr-qc]

[20] P. Cañate, P. Pearle, and D. Sudarsky, Phys.Rev. D 87, 104024 (2013), arXiv:1211.3463 [gr-qc].

[21] R. Penrose, Gen.Rel.Grav. 28, 581 (1996)

[22] L. Diosi, Phys.Lett. A120, 377 (1987).

[23] L. Diosi, Phys.Rev. A40, 1165 (1989). 
[24] G. Ghirardi, A. Rimini, and T. Weber, Phys.Rev. D34, 470 (1986),

[25] P. M. Pearle, Phys.Rev. A39, 2277 (1989).

[26] A. Bassi and G. C. Ghirardi, Phys.Rept. 379, 257 (2003), arXiv:quant-ph/0302164 [quant-ph].

[27] A. Bassi, K. Lochan, S. Satin, T. P. Singh, and H. Ulbricht, Reviews of Modern Physics 85, 471 (2013), arXiv:1204.4325 [quant-ph].

[28] L. Grishchuk and Y. Sidorov, Phys. Rev. D 42, 3413 (1990)

[29] L. Grishchuk, H. A. Haus, and K. Bergman, Phys. Rev. D46, 1440 (1992)]

[30] D. Polarski and A. A. Starobinsky, Classical and Quantum Gravity 13, 377 (1996), gr-qc/9504030.

[31] J. Lesgourgues, D. Polarski, and A. A. Starobinsky, Nucl. Phys. B497, 479 (1997), arXiv:gr-qc/9611019 [gr-qc]

[32] L. P. Grishchuk and J. Martin, Phys. Rev. D56, 1924 (1997), arXiv:gr-qc/9702018 [gr-qc].

[33] C. Kiefer and D. Polarski, Adv. Sci. Lett. 2, 164 (2009), arXiv:0810.0087 [astro-ph].

[34] C. Kiefer, I. Lohmar, D. Polarski, and A. A. Starobinsky, Class. Quant. Grav. 24, 1699 (2007), arXiv:astro-ph/0610700 [astro-ph]

[35] I. L. Egusquiza, A. Feinstein, M. A. Perez Sebastian, and M. A. Valle Basagoiti, Class. Quant. Grav. 15, 1927 (1998), arXiv:gr-qc/9709061 [gr-qc].

[36] C. P. Burgess, R. Holman, and D. Hoover, Phys. Rev. D77, 063534 (2008), arXiv:astro-ph/0601646 [astro-ph],

[37] A. Albrecht, P. Ferreira, M. Joyce, and T. Prokopec, Phys. Rev. D50, 4807 (1994), arXiv:astro-ph/9303001 [astro-ph].

[38] Y. Nomura, JHEP 11, 063 (2011), arXiv:1104.2324 [hep-th]

[39] Y. Nomura, Found. Phys. 43, 978 (2013), arXiv:1110.4630 [hep-th]

[40] V. Mukhanov, Physical Foundations of Cosmology (Cambridge University Press, New York, 2005).

[41] A. Valentini, (2008), arXiv:0804.4656 [hep-th].

[42] N. Pinto-Neto, G. Santos, and W. Struyve, Phys. Rev. D85, 083506 (2012), arXiv:1110.1339 [gr-qc].

[43] M. Benetti, S. J. Landau, and J. S. Alcaniz, JCAP 1612, 035 (2016), arXiv:1610.03091 [astro-ph.CO]

[44] A. Diez-Tejedor, G. Leon, and D. Sudarsky, Gen.Rel.Grav. 44, 2965 (2012), arXiv:1106.1176 [gr-qc]]

[45] J. Martin, V. Vennin, and P. Peter, Phys.Rev. D86, 103524 (2012), arXiv:1207.2086 [hep-th],

[46] S. Das, K. Lochan, S. Sahu, and T. Singh, Phys.Rev. D88, 085020 (2013), arXiv:1304.5094 [astro-ph.CO].

[47] S. Das, S. Sahu, S. Banerjee, and T. P. Singh, Phys. Rev. D90, 043503 (2014), arXiv:1404.5740 [astro-ph.CO].

[48] G. León and G. R. Bengochea, Eur. Phys. J. C76, 29 (2016), arXiv:1502.04907 [gr-qc].

[49] M. Mariani, G. R. Bengochea, and G. León, Phys. Lett. B752, 344 (2016), arXiv:1412.6471 [gr-qc],

[50] S. Banerjee, S. Das, K. Sravan Kumar, and T. P. Singh, Phys. Rev. D95, 103518 (2017), arXiv:1612.09131 [astro-ph.CO].

[51] S. Alexander, D. Jyoti, and J. Magueijo, Phys. Rev. D94, 043502 (2016), arXiv:1602.01216 [gr-qc].

[52] G. León, L. Kraiselburd, and S. J. Landau, Phys. Rev. D92, 083516 (2015), arXiv:1509.08399 [gr-qc]

[53] G. León, A. Majhi, E. Okon, and D. Sudarsky, Phys. Rev. D96, 101301 (2017), arXiv:1607.03523 [gr-qc]

[54] G. León, A. Majhi, E. Okon, and D. Sudarsky, Phys. Rev. D 98, 023512 (2018), arXiv:1712.02435 [gr-qc].

[55] C.-I. Kuo and L. H. Ford, Phys. Rev. D47, 4510 (1993), arXiv:gr-qc/9304008 [gr-qc],

[56] R. H. Brandenberger, H. Feldman, and V. F. Mukhanov, in Evolution of the universe and its observational quest. Proceedings, 37th Yamada Conference, Tokyo, Japan, June 8-12, 1993 (1993) pp. 19-30, arXiv:astro-ph/9307016 [astro-ph].

[57] K. Eppley and E. Hannah, Foundations of Physics 7, 51 (1977).

[58] J. Mattingly, Phys. Rev. D73, 064025 (2006), arXiv:gr-qc/0601127 [gr-qc].

[59] N. Huggett and C. Callender, Philosophy of Science 68, S382 (2001).

[60] M. Albers, C. Kiefer, and M. Reginatto, Phys. Rev. D78, 064051 (2008), arXiv:0802.1978 [gr-qc]

[61] A. Kent, "Simple Refutation of the Eppley-Hannah argument," (2018), arXiv:1807.08708 [gr-qc].

[62] D. N. Page and C. D. Geilker, Phys. Rev. Lett. 47, 979 (1981)

[63] S. Carlip, Micro and macro structure of spacetime. Proceedings, 11th and 12th Peyresq Physics Workshop, Peyresq, France, June 17-23, 2006 and June 16-22, 2007, Class. Quant. Grav. 25, 154010 (2008), arXiv:0803.3456 [gr-qc]

[64] D. Sudarsky, Proceedings, Relativity and Gravitation: Perspectives 100 years after Einstein's stay in Prague: Prague, Czech Republic, June 25-29, 2012, Fundam. Theor. Phys. 177, 349 (2014)

[65] A. Tilloy and L. Diósi, Phys. Rev. D93, 024026 (2016), arXiv:1509.08705 [quant-ph]

[66] V. F. Mukhanov, H. A. Feldman, and R. H. Brandenberger, Phys. Rept. 215, 203 (1992).

[67] G. León, G. R. Bengochea, and S. J. Landau, European Physical Journal C 76, 407 (2016), arXiv:1605.03632 [gr-qc].

[68] P. A. R. Ade et al. (Planck), (2015), arXiv:1502.01589 [astro-ph.CO].

[69] Planck Collaboration, N. Aghanim, M. Arnaud, M. Ashdown, J. Aumont, C. Baccigalupi, A. J. Banday, R. B. Barreiro, J. G. Bartlett, N. Bartolo, and et al., A\&A 594, A11 (2016), arXiv:1507.02704

[70] A. Lewis, A. Challinor, and A. Lasenby, APJ 538, 473 (2000).

[71] A. Lewis and S. Bridle, Phys. Rev. D66, 103511 (2002), arXiv:astro-ph/0205436 [astro-ph].

[72] F. Feroz, M. P. Hobson, and M. Bridges, Mon. Not. Roy. Astron. Soc. 398, 1601 (2009), arXiv:0809.3437 [astro-ph]

[73] F. Feroz and M. P. Hobson, Mon. Not. Roy. Astron. Soc. 384, 449 (2008) arXiv:0704.3704 [astro-ph],

[74] F. Feroz, M. P. Hobson, E. Cameron, and A. N. Pettitt, (2013), arXiv:1306.2144 [astro-ph.IM]

[75] E. Cameron and A. Pettitt, (2013), arXiv:1301.6450 [stat.ME]

[76] F. Beutler, C. Blake, M. Colless, D. H. Jones, L. Staveley-Smith, L. Campbell, Q. Parker, W. Saunders, and F. Watson, Mon. Not. Roy. Astron. Soc. 416, 3017 (2011), arXiv:1106.3366 [astro-ph.CO]

[77] A. J. Ross, L. Samushia, C. Howlett, W. J. Percival, A. Burden, and M. Manera, Mon.Not.Roy.Astron.Soc. 449, 835 (2015), arXiv:1409.3242

[78] L. Anderson et al. (BOSS), Mon. Not. Roy. Astron. Soc. 441, 24 (2014), arXiv:1312.4877 [astro-ph.CO] 
[79] R. Trotta, Mon. Not. Roy. Astron. Soc. 378, 72 (2007), arXiv:astro-ph/0504022 [astro-ph]

[80] M. Benetti and J. S. Alcaniz, Phys. Rev. D94, 023526 (2016) arXiv:1604.08156 astro-ph.CO].

[81] M. Benetti, S. J. Landau, and J. S. Alcaniz, JCAP 1612, 035 (2016), arXiv:1610.03091 [astro-ph.CO]

[82] L. L. Graef, M. Benetti, and J. S. Alcaniz, JCAP 1707, 013 (2017), arXiv:1705.01961 [astro-ph.CO]

[83] A. Heavens, Y. Fantaye, E. Sellentin, H. Eggers, Z. Hosenie, S. Kroon, and A. Mootoovaloo, (2017), arXiv:1704.03467 [astro-ph.CO].

[84] M. Campista, M. Benetti, and J. Alcaniz, (2017), arXiv:1705.08877 [astro-ph.CO].

[85] H. Jeffreys, Theory of probability (3rd edn. Oxfor University Press, First published in the Oxford Classics series 1998).

[86] R. Trotta, Mon. Not. Roy. Astron. Soc. 378, 72 (2007), arXiv:astro-ph/0504022 [astro-ph]

[87] A. G. Riess, L. Macri, S. Casertano, M. Sosey, H. Lampeitl, H. C. Ferguson, A. V. Filippenko, S. W. Jha, W. Li, R. Chornock, and D. Sarkar, Astrophysical Journal 699, 539 (2009), arXiv:0905.0695 [astro-ph.CO]

[88] J. L. Bernal, L. Verde, and A. G. Riess, JCAP 1610, 019 (2016), arXiv:1607.05617 [astro-ph.CO].

[89] M. Benetti, L. L. Graef, and J. S. Alcaniz, JCAP 1704, 003 (2017), arXiv:1702.06509 [astro-ph.CO]

[90] X. Zhang, Sci. China Phys. Mech. Astron. 60, 060421 (2017) arXiv:1702.05010 [astro-ph.CO].

[91] G. E. Addison, D. J. Watts, C. L. Bennett, M. Halpern, G. Hinshaw, and J. L. Weiland, (2017), arXiv:1707.06547 [astro-ph.CO]

[92] G. C. Carvalho, A. Bernui, M. Benetti, J. C. Carvalho, and J. S. Alcaniz, Phys.Rev.D 93, 023530 (2016), arXiv:1507.08972. 